\title{
A microRNA program controls the transition of cardiomyocyte hyperplasia to hypertrophy and stimulates mammalian cardiac regeneration
}

\author{
${ }^{1}$ Andrea Raso; ${ }^{1}$ Ellen Dirkx; ${ }^{1}$ Vasco Sampaio-Pinto; ${ }^{1,2}$ Hamid el Azzouzi; ${ }^{3,4}$ Ryan J. \\ Cubero; ${ }^{5}$ Daniel W. Sorensen; ${ }^{1}$ Lara Ottaviani; ${ }^{1}$ Servé Olieslagers; ${ }^{6}$ Manon M. Huibers; \\ ${ }^{6}$ Roel de Weger; ${ }^{7}$ Sailay Siddiqi; ${ }^{8}$ Silvia Moimas; ${ }^{8}$ Consuelo Torrini; ${ }^{8}$ Lorena Zentillin; 8 \\ Luca Braga; 1,9 Paula A. da Costa Martins; ${ }^{5}$ Jop H. van Berlo; ${ }^{8}$ Serena Zacchigna; 8,10 \\ Mauro Giacca and ${ }^{1}$ Leon J. De Windt*
}

${ }^{1}$ Department of Molecular Genetics, Faculty of Science and Engineering, Faculty of Health, Medicine and Life Sciences, Maastricht University, 6229 ER Maastricht, The Netherlands;

2Department of Molecular Genetics, Erasmus University MC, 3015 GD Rotterdam, The Netherlands;

${ }^{3}$ The Abdus Salam International Centre for Theoretical Physics, 34151 Trieste, Italy; ${ }^{4}$ Centre for Neural Computation, Kavli Institute for Systems Neuroscience, Norwegian University of Science and Technology, 7030 Trondheim, Norway

${ }^{5}$ Stem Cell Institute and Lillehei Heart Institute, Department of Medicine, University of Minnesota, Minneapolis, U.S.A.

${ }^{6}$ Department of Pathology, University Medical Center Utrecht, 3584 CX Utrecht, The Netherlands;

${ }^{7}$ Department of Cardiothoracic Surgery, Radboud University Medical Center, Nijmegen, the Netherlands;

8 International Centre for Genetic Engineering and Biotechnology (ICGEB), Trieste, Italy; ${ }^{9}$ Department of Physiology and Cardiothoracic Surgery, Faculty of Medicine, University of Porto, Porto, Portugal.

${ }^{10}$ School of Cardiovascular Medicine and Sciences, King's College London, London, UK.
A.R. and E.D. contributed equally to this work
* Correspondence should be addressed to L.D.W.

Manuscript information:

Introductory paragraph: 180 words

Body text: 3,625 words (without References)

Methods: 3,226 words

62 References

6 Main Figures, 6 Supplemental Figures, 4 Tables 
Myocardial regeneration is restricted to early postnatal life, when mammalian cardiomyocytes still retain the ability to proliferate. The molecular cues that induce cell cycle arrest of neonatal cardiomyocytes towards terminally differentiated adult heart muscle cells remain obscure. Here we report that the miR-106b 25 cluster is higher expressed in the early postnatal myocardium and decreases in expression towards adulthood, especially under conditions of overload, and orchestrates the transition of cardiomyocyte hyperplasia towards cell cycle arrest and hypertrophy by virtue of its targetome. In line, gene delivery of $\operatorname{miR}-106 b \sim 25$ to the mouse heart provokes cardiomyocyte proliferation by targeting a network of negative cell cycle regulators including E2f5, Cdkn1c, Ccne1 and Wee1. Conversely, gene-targeted miR-106b 25 null mice display spontaneous hypertrophic remodeling and exaggerated remodeling to overload by derepression of the prohypertrophic transcription factors Hand2 and Mef2d. Taking advantage of the regulatory function of $m i R-106 b \sim 25$ on cardiomyocyte hyperplasia and hypertrophy, viral gene delivery of $m i R-106 b \sim 25$ provokes nearly complete regeneration of the adult myocardium after ischemic injury. Our data demonstrate that exploitation of conserved molecular programs can enhance the regenerative capacity of the injured heart.

Proliferation of cardiogenic precursor cells and division of immature cardiomyocytes is key to mammalian cardiac morphogenesis during embryonic and fetal development. ${ }^{1,2}$ The neonatal mammalian heart still retains considerable proliferative capacity reminiscent of lower vertebrates, ${ }^{3-7}$ and is sustained by hypoxic conditions, ${ }^{8}$ activation of Hippo signaling, ${ }^{9}$ various transcriptional regulators, ${ }^{10-12}$ and endogenous microRNA mechanisms. ${ }^{13,14}$ Complete regeneration of the injured myocardium, including the human neonatal heart, ${ }^{15}$ exclusively takes place in a short time window during fetal and early postnatal life, when cardiomyocytes still possess the ability to proliferate. ${ }^{7}$ Shortly after birth, however, the majority of cardiomyocytes lose their proliferative capacity, and shift towards a terminally differentiated phenotype, with restricted ability to re-activate mitosis. ${ }^{16}$ Limited myocyte turnover does occur in the adult mammalian heart, but insufficient to restore contractile function following injury. ${ }^{17}$ The default growth response of the adult heart to overload or injury consists primarily of cardiomyocyte hypertrophy, a form of cellular growth without cell division that often precipitates in heart failure ${ }^{18}$ a serious clinical disorder that represents a primary cause of morbidity and 
hospitalization. In humans, despite remarkable progresses made by device-based therapies and drug interventions, ${ }^{19}$ the only way to replace lost cardiomyocytes is heart transplantation. Identification of the developmental molecular mechanisms that stimulate a proliferative phenotype in early postnatal life, control cell cycle arrest and produce a hypertrophic response of the terminally differentiated heart muscle, may hold the key to unlock the regenerative potential of the adult mammalian heart.

\section{RESULTS}

\section{Suppression of the miR-106b 25 cluster facilitates cardiac remodeling}

We recently demonstrated that mature miR-25 transcripts are downregulated in two mouse models of heart failure, and results in the derepression of the bHLH transcription factor Hand2 in the postnatal mammalian myocardium. ${ }^{20}$ miR-25 is embedded in the miR-106b 25 cluster, located on mouse chromosome 5 (chromosome 7 in humans) in an intronic region of the DNA-replication gene Mcm7 and consists of three miRNAs: $m i R-106 b$, miR-93 and miR-25 (Fig.1a). Here we show that each miR106b 25 cluster member displayed decreased expression in human cardiac biopsies of end-stage heart failure obtained upon heart transplantation compared to healthy controls (Fig.1b), decreased expression in the calcineurin transgenic mouse model of heart failure (Suppl.Fig.1a) and in pressure overloaded mouse hearts in both the early and late remodeling phase (Suppl.Fig.1b,c).

To more directly address the ramifications of reduced expression of $m i R$ 106b 25 for the postnatal heart, we subjected cohorts of miR-106b 25 -/- mice ${ }^{21}$, where expression of the cluster members was undetectable in the heart (Suppl.Fig.1d), to sham surgery or pressure overload by transverse aortic constriction (TAC) surgery and serially assessed cardiac geometry and function at four weeks (Fig.1c). Remarkably, miR-106b 25 -/- mice already suffered from a mild form of eccentric hypertrophy at baseline as evidenced in sham-operated mice by an increased cardiac geometry, reduced wall thickness, reduced ejection fraction (EF), and increased cardiomyocyte size at four weeks after sham surgery (Fig.1d-k; Table1). Four weeks of TAC surgery in wild-type mice or miR-106b 25 -/- mice resulted in severe myocyte disarray, interstitial fibrosis, increased heart weight, left ventricular dilation, systolic and diastolic dysfunction as well as a transcript induction of Nppa, Nppb, Acta1 and Myh7 as hypertrophic "stress" markers with consistently more severe phenotypes in miR-106b 25 -/- mice (Fig.1d-k; 
Table1). In line, silencing of the individual miR-106b 25 cluster members with specific antagomirs (Suppl.Fig.2a,b) resulted in spontaneous and mild eccentric remodeling as evidenced by an increased cardiac geometry (Suppl.Fig.2c), abnormal echocardiographic parameters (Suppl.Fig.2d-g), increased cardiomyocyte size (Suppl.Fig.2h) and induction of hypertrophic markers (Suppl.Fig.2j), albeit milder compared to the phenotype of miR-106b 25 -/- mice, supporting the conclusion that the individual cluster members work in a concerted fashion.

Mechanistically, we have previously shown miR-25-dependent regulation of the pro-hypertrophic embryonic transcription factor Hand2 in the adult heart and could repeat this finding (Suppl.Fig2k,I). ${ }^{20}$ To further understand the mechanistic role of $m i R$ 106b 25 in cardiac remodeling, we analyzed bioinformatics databases to search for miR-106b, miR-93 and miR-25 binding sites in cardiac expressed protein-coding transcripts. We identified a perfect match for a miR-25 heptametrical seed sequence in the transcription factor Myocyte enhancer factor $2 d(M e f 2 d)$ that showed complete evolutionary conservation among vertebrates (Fig.1I), validated the target gene using luciferase reporters harboring either the wild-type or site-directed mutagenesis of key nucleotides in the miR-25 binding site (Fig.1m), and finally demonstrated derepression of Mef2d protein expression in hearts from miR-106b 25 -/- mice in vivo by Western blotting (Fig.1n). Taken together, these data demonstrate that the miR-106b 25 cluster is repressed in the failing heart and causes eccentric hypertrophic remodeling by simultaneous derepression of the prohypertrophic transcription factors Hand2 and Mef2d.

\section{Activation of the miR-106b 25 cluster provokes cardiac enlargement}

To further evaluate the function of the miR-106b 25 cluster in cardiac disease, we measured the expression of $m i R-106 b, m i R-93$ and $m i R-25$ in the postnatal mouse heart at postnatal day 0 (p0), p5, p10, p15, and p20, representing developmental stages towards adulthood (p56, week 8). The data demonstrate that miR-106b 25 cluster was on average 6-8 fold higher expressed early in the postnatal phase and slowly decreased in expression to adult expression levels after which expression remained stable (Fig.2a). In line, miR-106b 25 expression in the adult heart muscle cells was very low compared to adult non-myocyte cells that still retain the ability to proliferate (Suppl.Fig.3). 
Next, we made use of the high cardiac tropism and prolonged expression of serotype 9 adeno-associated viral (AAV9) vectors following systemic delivery. ${ }^{22}$ AAV9 vectors expressing mmu-miR-106b, mmu-miR-93 and mmu-miR-25 precursor miRNAs (AAV9-miR-106b 25), or a control vector with an empty multiple cloning site (AAV9MCS), were injected intraperitoneally in neonatal mice at postnatal day 1 (p1; Fig.2b) as a gain-of-function approach (Fig.2c). Anticipating a cardiac phenotype resistant to hypertrophic remodeling, much to our surprise, at 4 weeks, the hearts of mice injected with AAV9-miR-106b 25 were histologically normal but significantly enlarged (Fig.2d,e). Echocardiographic analysis demonstrated that heart size and interventricular septum thickness in systole was significantly increased (Fig.2e-i, Table 2), but cardiac morphometric dimensions and contractile function were normal as evidenced by the absence of ventricular dilation (Fig.2h) and ejection fraction (Fig.2i). There was no sign of inflammatory cell infiltration or cardiac fibrosis (Fig.2d,j). Even more peculiar, individual myocyte size was unaltered and there was no evidence for reactivation of a "fetal" gene expression pattern characteristic for hypertrophic cardiomyocytes (Fig.2k,I). Conclusively, maintaining high miR-106b 25 expression levels as observed in the early postnatal developmental period produced cardiac enlargement in the adult heart without classical signs of pathological hypertrophic remodeling.

\section{The miR-106b 25 cluster stimulates cardiomyocyte proliferation}

To understand how the miR-106b 25 cluster can evoke cardiac growth, we resorted to cardiomyocyte cultures isolated from neonatal hearts, which retain both hypertrophic and proliferative properties. ${ }^{23}$ We performed a fluorescence-microscopybased analysis in neonatal rat cardiomyocytes transfected with either a control precursor miRNA, or precursors for miR-106b, miR-93 or miR-25. At $72 \mathrm{~h}$, cells were stained for sarcomeric a-actinin to distinguish cardiomyocytes from non-myocytes, and we included the proliferation marker 5-ethynyl-2'-deoxyuridine (EdU), a thymidine analogue that is incorporated into newly synthesized DNA (Fig.3a,b). Automated image segmentation and analysis was performed to selectively quantify number of proliferating cardiomyocytes (a-actinin+, EdU+; Fig.3c) and total number of cardiomyocytes (aactinin+; Fig.3d; Suppl.Fig.4a). The data demonstrate that $m i R-106 b, m i R-93$ or $m i R-25$ stimulated cardiomyocyte proliferation and cardiomyocyte numbers by a factor of 3 with no substantial differences between the miRNA cluster members. 
Next, to evaluate whether the miR-106b 25 cluster would also enhance cardiomyocyte proliferation in vivo, we injected AAV9-miR-106b 25 intraperitoneally in neonatal mice at $\mathrm{p} 1$ to elevate cardiac miR-106b 25 cluster expression, administered Edu intraperitoneally at p10 and analyzed the hearts at p12 (Fig.3e). The hearts of p12 mice injected with AAV9-106b 25 were significantly enlarged compared to those from mice injected with the control AAV9 (Fig.3f), with no sign of inflammatory cell infiltration or increased cardiac fibrosis content, and no increase in cardiomyocyte size (Fig.3f,g). Confocal microscopy indicated that the number of both cardiomyocytes in $\mathrm{S}$ phase of the cell cycle (a-actinin+, EdU+; Fig.3h, I; Suppl.Fig.1e) and mitotic cardiomyocytes (aactinin+, PH3+; Fig.3h,j; a-actinin+, Aurora B+; Suppl.Fig.4b) was significantly increased in hearts of animals injected with AAV9-miR-106b 25 compared to hearts of animals injected with the control AAV9-MCS.

Next, to ascertain if the individual cluster members showed differences in activating cardiomyocyte proliferation, we also generated AAV9 vectors expressing either miR-106b, miR-93 or miR-25 and administered the viral vectors intraperitoneally in neonatal mice at p1, administered EdU at p10 and analyzed the hearts at p12 (Suppl.Fig.5a). In each case, mice with increased expression of either miR-106b, miR93 or miR-25 were significantly enlarged (Suppl.Fig.5b), showed no increase in cardiomyocyte cell size (Suppl.Fig.5b,c), demonstrated an increased number of cardiomyocytes in S phase of the cell cycle (a-actinin+, EdU+; Suppl.Fig.5d,e) and mitotic cardiomyocytes (a-actinin+, PH3+; Suppl.Fig.5f,g; or a-actinin+, Aurora B+; Suppl.Fig.5h).

Next, we employed stereology to simultaneously assess left ventricular volumes, $\mathrm{CM}$ volumes, CM nuclei densities, CM nucleation and CM proliferation as described previously24-26 in wild-type neonatal mice, wild-type mice injected with AAV9-miR106b 25, and miR-106b 25 knockout mice from birth (p0) to p12, since CM proliferation is still abundant in early postnatal life in the mouse and essentially absent after p15 ${ }^{24}$ (Fig.4a). CM nuclei in tissue sections were stained for the cardiomyocyte nuclear marker pericentriolar material 1 (PCM-1), CM multinucleation was determined by co-staining with wheat germ agglutinin (WGA) to delineate cell boundaries and PCM-1 to mark CM nuclei and CM proliferation assessed by co-labelling sections with PCM-1 and EdU (Fig.4b). LV volumes increased from $5.5 \pm 0,3 \mathrm{~mm}^{3}$ to $13.2 \pm 0.8 \mathrm{~mm}^{3}$ at $\mathrm{p} 6$ and $\mathrm{p} 12$, respectively, with a tendency for mice that received AAV9-miR-106b 25 to have a 
slightly higher LV volume (Fig.4c). The ratio of mono- to binucleated cardiomyocytes changed substantially during the first 12 postnatal days. On p6, a minority of cardiomyocytes $(14.1 \pm 1.1 \%)$ were binucleated, whereas at p12 the majority of cardiomyocytes became binucleated $(66.1 \pm 0.9 \%)$, with mice that received AAV9-miR106b 25 having a slight but significant reduction in the percentage of binucleated cardiomyocytes at p12 (61.2 2 2.0\%; Fig.4d). The total number of cardiomyocytes at p12 was considerably larger in mice that received AAV9-miR-106b 25 as were the number of EdU+ cardiomyocytes at p6 and p12 (Fig.4e,f). In addition, the number of EdU+ cardiomyocytes was lower at p12 in miR-106b 25 knockout mice, indicating a requirement of the endogenous miR-106b 25 cluster for cardiomyocyte proliferation in juvenile hearts (Fig.4f).

Taken together, these results show that elevated cardiac expression of the complete miR-106b 25 cluster or the individual cluster members significantly enhance proliferation of at least a subset of cardiomyocytes in vivo.

\section{The miR-106b 25 cluster suppress cell cycle inhibitors}

To elucidate the molecular mechanisms underlying the proliferative effects of this microRNA cluster, we performed RNA-seq to assess the transcriptome changes in neonatal rat cardiomyocyte RNA after transfection with miR-106b, miR-93 or miR-25 mimics. This analysis identified 1,082 genes for $m i R-106 b, 1,347$ genes for $m i R-93$, and 1,673 genes for miR-25 upregulated and 1,253 genes for miR-106b, 1,594 genes for miR-93, and 2,327 genes for miR-25 downregulated (at 1.0 reads per kilobase of exon model per million mapped reads (RPKM) cutoff and 1.30 fold-change cutoff; Fig. 5a, Suppl.TableS1). We then imposed bioinformatic predictions of miRNA seed sequence interactions with rat transcripts that were downregulated by the miRNAs upon transfection to cardiomyocytes according to the transcriptomic data, yielding 112, 217, and 420 miR-106b, miR-93 and miR-25 target genes respectively, with substantial overlap between the miR-106b and miR-93 targetome (Fig. 5b). Next, a bioinformatic protein-protein interaction network was derived that integrates and scores protein interactions across different evidence channels (conserved neighborhood, cooccurrence, fusion, co-expression, experiments, databases and text mining). For each miRNA in the miR106b 25 cluster, a subnetwork was extracted. Analysis of the networks showed a particularly dense and often overlapping enrichment for genes 
functioning in cell cycle regulation, and to a lesser extent networks of genes involved in actin cytoskeletal organization, oxidative stress, and components of the Hippo pathway (Fig. 5c).

miR-106b and miR-93 had overlapping targets among the cyclins and cyclindependent kinases including Ccnb1 (cyclin B), Ccna2 (cyclin A), Ccnd1 and Ccnd2 (cyclin Ds) as well as Ccne2 (cyclin E), suggesting that these two miRNAs affected the G2-phase of the cell cycle (Fig 5d). miR-25 showed an upregulation of Ccnd1 and Ccnd2 (cyclin Ds) accompanied by a downregulation of Ccne1 and Ccne2 (cyclin E), Ccna2 (cyclin A) and Ccnb1 (cyclin B), suggesting that this miRNA affected the S-phase of the cell cycle (Fig 5d). Apart from regulation of specific Cyclin/Cdk complexes, common targets among all members of the miR-106b 25 cluster included various cell cycle inhibitors that act on various cell cycle phases, including Cdkn1a (cyclin-dependent kinase inhibitor 1a or p21 ${ }^{\mathrm{CIP} 1}$ ), Cdkn1c (cyclin-dependent kinase inhibitor 1c or p57 ${ }^{\mathrm{KIP} 2}$ ), the retinoblastoma transcriptional corepressor $1(\mathrm{Rb} 1)$ inhibitor E2F5, and the $\mathrm{G} 2$ checkpoint kinase Wee1 (Fig 5c,d).

We independently validated our results with an unbiased high-content screen in neonatal rat cardiomyocytes transfected with siRNAs against $18 \mathrm{miR}-106 \mathrm{~b} 25$ targetome members to screen for their individual contribution to cardiomyocyte proliferation. The data demonstrate that treatment siRNAs against individual targetome members induced only a partial increase in CM proliferation compared to that observed with miR-106b 25 overexpression, indicating that the effect of the miRNA cluster probably results from a cumulative effect on multiple, cellular mRNA targets (Fig.5e,f). Finally, we identified perfect matches for the heptametrical seed sequence for the miRNAs in the 3'UTRs of E2F5 and Cdkn1c that showed evolutionary conservation among vertebrates (Suppl.Fig.6a,c), validated the target genes using luciferase reporters harboring either the wild-type or site-directed mutagenesis of key nucleotides in the miRNA binding sites (Suppl.Fig.6b,d), and finally demonstrated derepression of E2F5, Cdkn1c, Wee1 and Ccne1 protein expression in cultured cardiomyocytes transfected with an antimiR for miR-106b, miR-25 or in hearts from miR-106b 25 null mice by Western blotting (Suppl.Fig.6e-g).

Collectively, these data demonstrate that the miR-106b 25 cluster regulates densily overlapping networks of genes involved in cell cycle regulation and a number of key cell cycle inhibitors, explaining the proliferative effects of this miRNA cluster. 


\section{The $m i R-106 b \sim 25$ cluster stimulates post-infarction cardiac regeneration}

The adult heart is characterized by a very poor regenerative potential. Given that the miR-106b 25 cluster is higher expressed in the early postnatal phase and regulates cell cycle regulators and postnatal cardiomyocyte proliferation, we hypothesized that viral delivery of the miRNA cluster could potentially enhance post-infarction regeneration in the adult heart. To test this, adult CD1 mice underwent permanent ligation of left anterior descending (LAD) coronary artery to induce myocardial infarction (MI) and hearts were injected in the peri-infarcted area with AAV9-miR-106 25 or a control AAV9 vector (AAV-MCS) (Fig.6a). This approach resulted in efficient overexpression of each miRNA over control expression levels after MI (Fig.6b). At three weeks of MI, crosssectioning hearts from ligation to base demonstrated that hearts injected with the control AAV9 vector displayed the typical large and thinned scarred infarct accompanied with severe biventricular dilation (Fig.6c,d). In sharp contrast, hearts injected with AAV9-miR106 25 demonstrated significantly reduced infarct size and preservation of viable LV tissue and cardiac geometry (Fig.6c,d). Echocardiographic analysis demonstrated near complete normalization of LV mass (Table 3), LVPWd (Fig.6e), LVIDs (Fig.6f), LVEF (Fig.6g) and other functional parameters (Table 3), and a reduction in "stress" marker genes (Suppl.Fig.6h). Confocal microscopy revealed that mice that received AAV9-miR106b 25 at three weeks displayed a significant number of EdU-positive cardiomyocyte nuclei in the infarct zone (Fig.6h) with well-integrated cardiomyocytes within the myocardial structure indicative of active proliferation and regeneration following infarction.

Finally, we cross-bred tamoxifen-inducible genetic lineage-tracing Myh6MerCreMer mice to a Rosa26 tdTomato reporter mice to permanently mark Myh6expressing cells (i.e. cardiomyocytes) and follow the fate of their cellular descendants in vivo. Accordingly, adult Myh6-MCMR26tdTomato animals were treated with tamoxifen daily for 5 days to label cardiomyocytes with tdTomato. Next, Myh6-MCMR26tdTomato mice underwent permanent ligation of left anterior descending (LAD) coronary artery to induce $\mathrm{MI}$ and hearts were injected in the peri-infarcted area with AAV9-miR-106 25 or a control AAV9 vector. A week before sacrifice, all animals received Edu to mark nuclei that are in S phase of the cell cycle and we analyzed EdU+/tdTomato+ cells in tissue sections (Fig.6i). As expected, tdTomato was exclusively expressed in cardiomyocytes. 
Importantly, the number of EdU+/tdTomato+ cells in post-infarcted hearts that received AAV9-miR-106 25 was doubled compared to post-infarcted hearts that received a control AAV9 vector. This genetic lineage tracing approach confirms that the AAV9-miR106 25 vector stimulated proliferation of pre-existing cardiomyocytes (Fig 6i-k).

Conclusively, the miR-106b 25 cluster, relatively high expressed in the early postnatal myocardium that still retains regenerative potential, directs networks of cell cycle regulators and stimulates proliferation of at least a subset of cardiomyocytes in vivo. In adulthood, the relative low cardiac expression of miR-106b 25 sustains derepression of prohypertrophic cardiomyocyte gene programs that facilitate adverse remodeling in response to overload (Fig.6I). Exploiting this endogenous regulator between cardiomyocyte hyperplasia and hypertrophy by viral gene delivery enhances the endogenous regenerative capacity of the mammalian myocardium.

\section{DISCUSSION}

Upon after birth, cardiomyocytes enter cell cycle arrest and become terminally differentiated accompanied by polyploidy and hypertrophy as the default growth response to overload or injury.7,27,28 This terminally differentiated phenotype and reduced cellular plasticity makes the heart more vulnerable in situations when increased workload is required as it either triggers irreversible cell death or hypertrophy, ${ }^{29}$ which often precipitates in heart failure, a serious clinical disorder that represents the primary cause of morbidity and hospitalization in Western societies.

Here we report on the evolutionarily conserved microRNA cluster that is highly expressed in the early postnatal myocardium and repressed in the adult heart in man and mouse under disease conditions. Remarkably, miR-106b 25 deficient mice as well as mice receiving antagomirs for either $m i R-106 b, m i R-93$ or $m i R-25$, display spontaneous cardiomyocyte hypertrophy and eccentric remodeling, mechanistically explained by the derepression of prohypertrophic downstream targets, most notably the bHLH transcription factor Hand2,20 as well as myocyte enhancer factor-2d (Mef2d), which serves as a terminal branch of stress signaling pathways that drive pathological cardiac remodeling. ${ }^{30}$

Overexpression of the miR-106b 25 cluster, or the individual cluster members miR-106b, miR-93 or miR-25, by adeno-associated viral (AAV) vectors, stimulated cardiomyocyte proliferation, at least in a subset of cardiomyocytes, by targeting a 
network of genes with cell cycle regulatory functions including the key cell cycle inhibitors E2f5, Cdkn1c, Ccne1 and Wee1, positive cell cycle regulators that are abundantly expressed in the fetal and neonatal heart. ${ }^{31,32}$ In the adult heart, cyclindependent kinase inhibitors, negative regulators of the cell cycle, are more prevalent. ${ }^{31,32}$ In line, forced overexpression of cyclin D2, a positive regulator of the G1/S transition, induced DNA synthesis and proliferation in mammalian cardiomyocytes. ${ }^{31,32}$ Additionally, overexpression of cyclin A2, which promotes the G1/S and G2/M transitions, results in cardiomyocyte proliferation, ${ }^{33}$ improved cardiac function after ischemic injury in mice ${ }^{34}$ and pigs. ${ }^{35}$

That members of the miR-106b 25 cluster can evoke cardiomyocyte proliferation is confirmed by an unbiased, high-content screen to identify proliferative microRNAs, ${ }^{36}$ while more recently, miR-25 was demonstrated to provoke cardiomyocyte proliferation in zebrafish by repressing the cell cycle inhibitor Cdknc1 and tumor suppressor Lats2. ${ }^{37}$ Our results also revealed components of the Hippo/Yap pathway as miR-106b 25 targetome members. Hippo signaling has been widely studied in the context of cardiac regeneration. ${ }^{38,39}$ In line, embryonic overexpression of Yap in mice induces hyperproliferation of cardiomyocytes and severely disproportional ventricles and death, $38,40,41$ while forced expression of Yap in the adult heart provokes cardiomyocyte cell cycle re-entry and regeneration postinfarction injury. ${ }^{40,42}$ However, unrestrained Yap activation may also display unwanted effects in pressure overloaded hearts due to cardiomyocyte dedifferentiation. ${ }^{43}$

Interestingly, contradicting effects of $m i R-25$ in the rodent heart have been reported. Some reports indicate that inhibition of $m i R-25$ expression can lead to derepression of the target gene Serca2a and improve cardiac function, ${ }^{44}$ and others report protection against oxidative stress or apoptosis induced by sepsis. ${ }^{45,46}$ In contrast, others report that overexpression of $m i R-25$ is innocuous and induces proliferation by altering cell cycle genes in zebrafish, ${ }^{37}$ while here we report cardiac enlargement secondary to enhanced cardiomyocyte proliferation, which at first sight could be misinterpreted as a pathological phenotype. From a therapeutic perspective, $m i R-25$ loss-of-function approaches have also shown disparate results from improving contractility on the one side, ${ }^{44}$ or inducing high blood pressure, ${ }^{47}$ atrial fibrillation, ${ }^{48}$ eccentric remodeling and dysfunction, ${ }^{20,47}$ on the other. It should be noted that distinct chemistries of antisense oligonucleotides can show quite different specificity or even 
cause side-effects that may explain the opposing observations. ${ }^{49,50}$ To avoid the uncertainty surrounding the use of oligonucleotide chemistries, here we resorted to an unequivocal gene deletion strategy where miR-106b 25 null mice display pathological cardiomyocyte hypertrophy, fibrosis, cardiac dilation and dysfunction, phenotypes that were recapitulated when silencing the individual cluster members with a 2'Ome antisense chemistry. Using the same gene deletion approach, miR-106b 25 knockout mice show enhanced paroxysmal atrial fibrillation related to disruption of a paired-like homeodomain transcription factor 2 homeobox gene (Pitx2) driven mechanism that controls the expression of the miR-17 92 and miR-106b 25 clusters. ${ }^{51}$ In line, Pitx2 lies in close proximity to a major atrial fibrillation susceptibility locus on human chromosome 4q25 identified in genome-wide association studies. ${ }^{52}$ Taken together, exceptional scrutiny should be considered when designing silencing strategies to therapeutically intervene in miR-25 expression in heart disease.

The combined observations in this study suggest a model whereby defined orchestration of cell cycle regulators underlies the developmental cell cycle arrest of postnatal cardiomyocytes. Moreover, the characteristics of miR-106b 25 expression in this developmental time frame and its targetome provides a mechanistic explanation for cell cycle exit towards the acquirement of the terminally differentiated phenotype. Hence, when miR-106b 25 expression is higher, as is the case in the early postnatal heart, cell cycle inhibitors including E2f5, Cdkn1c, Ccne1 and Wee1 are actively suppressed resulting in a proliferative state and cardiomyocyte hyperplasia, while differentiation programs elicited by Hand2 and Mef2d are suppressed. Vice versa, in the overloaded or injured adult heart when miR-106b 25 expression is lower, cell cycle re-entry is actively suppressed by the derepression of cell cycle inhibitors, and a prohypertrophic terminal differentiation program is promoted. Taking advantage of the regulatory function between cardiomyocyte hyperplasia and hypertrophy by viral gene delivery of miR106b 25 produced regeneration of the adult myocardium in response to chronic ischemic injury. Our data demonstrate that exploitation of conserved epigenetic molecular programs can enhance the regenerative capacity of the injured myocardium. 


\section{FIGURE LEGENDS}

Figure 1 I miR-106b 25 gene deletion induces hypertrophic cardiac remodeling.

(a) A schematic representation of the mouse $M c m 7$ gene harboring the miR-106b 25 cluster in intron 13. (b) Real-time PCR analysis of miR-106b, miR-93 and miR-25 abundance in human non-failing or failing myocardium. (c) Design of the study. (d) Representative images of whole hearts (top panels), haematoxylin \& eosin (H\&E)stained sections of four-chamber view (second panel), high magnification H\&E sections (third panel), Sirius Red stained sections (fourth panel) and wheat germ agglutinin (WGA)-stained (fifth panel) histological sections. Quantification of (e) left ventricular mass/body weight (BW) ratio, (f) left ventricular posterior wall thickness in systole (LVPWs), (g) left ventricular internal diameter in systole (LVIDs), (h) ejection fraction (EF). (i) Quantification of the fibrotic area by Sirius Red staining and (j) cell surface areas by wheat germ agglutinin (WGA) staining. (k) Real-time PCR analysis of Nppa, Nppb, Acta1 and Myh7, $n$ refers to number of animals. (I) Location and evolutionary conservation of hsa-miR-25 seed region on Mef2d. $(\mathbf{m})$ Activity assay of luciferase reporter constructs shows the binding of $h s a-m i R-25$ to the 3'UTR of Mef2d, $n$ refers to number of transfection experiments. (n) Western blot analysis of endogenous Mef2d and GAPDH as a loading control in hearts from wild type (WT) versus miR-106b 25 knockout (KO) mice, $n$ refers to number of hearts. ${ }^{*} P<0.05$ vs corresponding control group; $\# P<0.05$ vs corresponding treatment (error bars are s.e.m.).

Figure 2 | Overexpression of the miR-106b 25 cluster induces cardiac growth with sustained function. (a) Real-time PCR analysis of miR-106b, miR-93 and miR-25 abundance in mouse hearts at postnatal day 0 (p0), p5, p10, p15, p20 and p56 (2 months) of age. (b) Design of the study. (c) Real-time PCR analysis of miR-106b, miR93 and miR-25 abundance in AAV9-MCS versus AAV9-miR-106b 25 hearts, 4 weeks after injection. (d) Representative images of whole hearts (top panels), H\&E-stained sections of four-chamber view (second panel), high magnification H\&E sections (third panel), Sirius Red stained sections (fourth panel) and WGA-stained (fifth panel) sections. Quantification of (e) LV/BW ratio, (f) LVPWs, (g) IVSs, (h) LVIDs, and (i) EF of mice that received AAV9-MCS or AAV9-miR-106b 25. Quantification of (j) the fibrotic 
area by Sirius Red staining and (k) the cell surface areas by WGA-staining. (I) Real-time PCR analysis of Nppa, Nppb, Acta1, and Myh7 in hearts from mice that received AAV9MCS or AAV9-miR-106b 25; or WT or calcineurin transgenic (Myh6-CnA) mice, a mouse model for heart failure, $n$ refers to number of animals. ${ }^{*} P<0.05$ vs corresponding control group (error bars are s.e.m.).

Figure 3 I Overexpression of the miR-106b 25 cluster induces cardiomyocyte (CM) proliferation. (a) Workflow of the experiment. (b) Representative fluorescent microscopic images of rat CMs transfected with a control precursor miRNA, or precursors for miR-106b, miR-93 or miR-25 and stained for a-actinin, 5-ethynyl-2'deoxyuridine (EdU), and Hoechst. Quantification of (c) the number of proliferating CMs (a-actinin+, EdU+) and (d) total number of CMs (a-actinin+) after transfection with a control miRNA, or miR-106b, miR-93 or miR-25, $n$ refers to the number of transfection experiments. (e) Workflow of the study. (f) Representative images of whole hearts (top panels), H\&E-stained sections in four-chamber view (second panel), Sirius Red stained sections (third panel) and WGA-stained (fourth panel) sections. (g) Quantification of the cell surface areas from WGA-stained sections. (h) Confocal microscopy images of heart sections of mice treated with AAV9-MCS or AAV9-miR-106b 25 and stained for aactinin, EdU and Hoechst or a-actinin, $\mathrm{pH} 3$ and Hoechst. Quantification of (i) the number of proliferating CMs (a-actinin+, EdU+) and (j) the number of phospho-histone $3(\mathrm{pH} 3)$ positive $\mathrm{CMs}$ (a-actinin+, $\mathrm{pH} 3+$ ), $n$ refers to number of hearts. ${ }^{*} P<0.05$ vs corresponding control group (error bars are s.e.m.).

Figure 4 I The miR-106b 25 cluster controls total number of CMs. (a) Workflow of the study. (b) Representative confocal images of sections co-stained for pericentriolar material 1 (PCM-1), 4',6-diamidino-2-phenylindole (DAPI), EdU and WGA to assess PCM-1+EdU+ CMs in the experimental groups at p12. Quantification of (c) LV volumes, (d) percentage CM binucleation, (e) total number of CMs and (f) proliferating (PCM$1+, \mathrm{EdU}+) \mathrm{CMs}$ at p6 and p12 in the experimental groups, $n$ refers to number of hearts. ${ }^{\star} P<0.05$ vs corresponding control group (error bars are s.e.m.).

Figure 5 I The miR-106b 25 cluster suppresses cell cycle inhibitors. (a) Workflow for the experiment and miRNA target validation. (b) Venn diagram showing the 
relationship between the sets of downregulated genes with miR-106b, miR-93 or miR-25 seed regions and a 1.3-fold change cut-off with respect to cel-miR-67. (c) A compartmentalized network of differentially expressed genes involved in cell cycle regulation. Each connection is color-coded according to the miRNA that regulates the differentially expressed gene (magenta for miR-106b, cyan for miR-93 and dark yellow for miR-25). Genes indicated by blue circles are downregulated by at least one miRNA while downregulated genes shown in blue squares are found to be bioinformatic targets by at least 1 miRNA. Genes represented by yellow circles are upregulated genes by all three miRNAs. (d) Heatmap representation of cell cycle regulators differentially expressed by miR-106b 25 relative to cel-miR-67. (e) EdU staining of rat CMs treated with selected siRNAs. (f) Percentage of proliferating rat CMs after siRNA treatment. ${ }^{*}<$ 0.05 vs corresponding control group (error bars are s.e.m.).

Figure 6 I The miR-106b 25 cluster evokes myocardial regeneration. (a) Design of the study. (b) Real-time PCR analysis of miR-106b, miR-93 and miR-25 abundance in infarcted hearts receiving AAV9-MCS or AAV9-miR-106b 25. (c) Representative images of Sirius Red-stained ventricular cross-sections from the point of ligation towards the apex of hearts post-MI, treated with AAV9-MCS or AAV9-miR-106b 25. (d) Quantification of the infarct area of the post-infarcted left ventricles from mice receiving AAV9-MCS or AAV9-miR-106b 25. Quantification of (e) LVPWd, (f) LVIDs and (g) EF of mice that received AAV9-MCS or AAV9-miR-106b 25 after sham or MI surgery. (i) Representative images of the infarct border zone of mice treated with AAV9-MCS or AAV9-miR-106b 25 after MI surgery, and stained for a-actinin and EdU; $n$ refers to the number of animals. (i) Design of the lineage tracing study. (j) TdTomato expression overlaps with 5-ethynyl-2'-deoxyuridine (EdU). (k) Quantification of TdTomato+, EdU+ CMs. (I) Schematic representation of the model. ${ }^{*} P<0.05$ vs corresponding control group; \#P<0.05 vs corresponding treatment group (error bars are s.e.m.).

\section{Supplemental Figure 1 I miR-106b 25 cluster expression. (a) Real-time PCR} analysis of $m i R-106 b$, miR-93 and miR-25 abundance in hearts from WT or Myh6-CnA mice (b) mice subjected to transverse aortic constriction (TAC) for 1, 2 or 6 weeks. (c) Indices of cardiac dilation (LVIDs), function (EF) and expression of Nppb and Myh7 in mice subjected to TAC. (d) Real-time PCR analysis of miR-106b, miR-93 and miR-25 
abundance in hearts of wild-type (WT) or miR-106b 25 null mice, $n$ refers to the number of hearts. ${ }^{*} P<0.05$ vs corresponding control group (error bars are s.e.m.).

Supplementary Figure 2 I miR-106b 25 silencing with antagomirs. (a) Workflow of the study. (b) Real-time PCR analysis of miR-106b, miR-93 and miR-25 expression in hearts from mice receiving control (ctrl) antagomir or antagomir against a specific miRNA. (c) (d) Representative images of whole hearts (top panels), H\&E-stained sections (second panel), Sirius Red stained sections (third panel) and WGA-stained (fourth panel) histological sections. Quantification of (d) LV/BW ratio, (e) LVPWs, (f) LVIDs, and (g) EF of mice that received indicated antagomirs. Quantification of (h) cell surface areas by WGA-staining and (i) fibrotic area by Sirius Red staining. (j) Real-time PCR analysis of Nppa, Nppb, Acta1, and Myh7; $n$ refers to number of hearts. (k) Location and evolutionary conservation of hsa-miR-25 seed region on Hand2. (I) Activity assay of luciferase reporter constructs shows the binding of hsa-miR-25 to the 3'UTR of Hand2, $n$ refers to number of transfection experiments. ${ }^{\star} P<0.05$ vs corresponding control group (error bars are s.e.m.).

\section{Supplemental Figure 3 I miR-106b 25 cluster expression in adult heart cells. (a)}

Real-time PCR analysis of marker genes for Tropomyosin 1 (Tpm1), Platelet endothelial cell adhesion molecule (Pecam1) and Transcription factor 21 (Tcf21) in CMs, ECs and FBs following enzymatic dissociation of adult mouse hearts followed by column-based magnetic cell isolation. (b) Real-time PCR analysis of miR-106b, miR-93 and miR-25 abundance in different heart cells, $n$ refers to the number of hearts.

\section{Supplemental Figure 4 I Overexpression of $m i R-106 b, m i R-93$ or $m i R-25$ stimulates} CM proliferation. (a) FACS analysis showing the percentage of a-actinin+, EdU+ CMs isolated from p12-old mice that received AAV9-MCS or AAV9-miR-106b 25. (b) Confocal microscopy images of heart sections of mice treated with AAV9-MCS or AAV9miR-106b 25 and stained for a-actinin, Aurora B and Hoechst.

\section{Supplemental Figure 5 I Overexpression of $m i R-106 b, m i R-93$ or $m i R-25$ induces cardiac enlargement by stimulating cardiomyocyte proliferation. (a) Design of the study. (b) Representative images of whole hearts (top panels), H\&E-stained histological}


sections of four-chamber view (second panel) and WGA-stained (third panel) histological sections. (c) Quantification of cell surface areas. (d) Representative confocal microscopy images and (e) quantification of proliferating cardiomyocytes (a-actinin+, EdU+) in heart sections of mice receiving AAV9-MCS, AAV9-miR-106b, AAV9-miR-93 or AAV9-miR-25 and stained for a-actinin, EdU and Hoechst. (f) Representative confocal microscopy images and $\mathbf{( g )}$ quantification of the number of $\mathrm{pH} 3$ positive $\mathrm{CMs}(\mathrm{a}$-actinin+, $\mathrm{pH} 3+)$ in heart sections of mice receiving AAV9-MCS, AAV9-miR-106b, AAV9-miR-93 or AAV9miR-25 and stained for a-actinin, $\mathrm{pH} 3$ and Hoechst, $n$ refers to number of hearts. (h) Representative confocal microscopy images of heart sections of mice receiving AAV9MCS, AAV9-miR-106b, AAV9-miR-93 or AAV9-miR-25 and stained for a-actinin, Aurora $B$ and Hoechst. ${ }^{\star} P<0.05$ vs corresponding control group (error bars are s.e.m.).

\section{Supplemental Figure 6 I miR-106b 25 targetome validation. (a) Location and} evolutionary conservation of the hsa-miR-106b seed region on E2f5. (b) Activity assay of luciferase reporter constructs shows the binding of hsa-miR-106b to the 3'UTR of E2f5. (c) Location and evolutionary conservation of the hsa-miR-25 seed region on Cdkn1c. (d) Activity assay of luciferase reporter constructs shows the binding of $h s a-m i R-25$ to the 3'UTR of Cdkn1c, $n$ refers to number of transfection experiments. (e) Western blot analysis of endogenous E2f5 and Cdkn1c and histone $3(\mathrm{H} 3)$ as a loading control in cardiomyocytes transfected with a control antimiR, or antimiRs for miR-106b or miR-25.

(f) Western blot analysis of endogenous Wee1, Ccne1 and Tubulin-a (Tuba1a) as a loading control in cardiomyocytes transfected with a control antimiR, or an antimiR for miR-106b. (g) Western blot analysis of endogenous E2f5, Cdkn1c, Ccne1 and Gapdh as a loading control in hearts from WT versus miR-106b 25 KO mice, $n$ refers to the number of animals. (h) Real-time PCR analysis of Nppa, Nppb, Acta1, and Myh7; $n$ refers to number of hearts. ${ }^{\star} P<0.05$ vs corresponding control group (error bars are s.e.m.). 


\section{METHODS}

Human heart samples. Approval for studies on human tissue samples was obtained from the Medical Ethics Committee of the University Medical Center Utrecht, The Netherlands, and by the Ethical Committee of the University Hospital Hamburg, Germany (Az. 532/ 116/9.7.1991). All patients or their relatives gave written informed consent before operation. In this study, we included tissue from the left ventricular free wall of patients with end-stage heart failure secondary to ischemic heart disease. Control tissue was taken from the left ventricular free wall of refused donor hearts. Failing hearts were also obtained from patients undergoing heart transplantation because of terminal heart failure. Non-failing donor hearts that could not be transplanted for technical reasons were used for comparison. The donor patient histories did not reveal any signs of heart disease.

Mouse models. Mice homozygous null for the mirc3 cluster (miR-106b 25) located in intron 13 of the $\mathrm{Mcm} 7$ (minichromosome maintenance complex component 7 ) gene were generated previously, ${ }^{21}$ obtained from the Jackson Laboratory (Mirc3tm1.1Tyj/J, Stock No: 008460) and maintained in a B6SV129F1 background. Both male and female miR$106 b \sim 25$ null mice of 3-6 months of age were used in this study. Other mice used in this study were 3-6 month old male calcineurin transgenic mice in a B6SV129F1 background expressing an activated mutant of calcineurin in the postnatal heart under control of the $5.5 \mathrm{~kb}$ murine myh6 promoter (MHC-CnA) ${ }^{53}$; male and female CD1 wild-type mice ranging between postnatal (p) day 0 and p56; male and female B6SV129F1 wild-type mice of 3-6 months of age (Charles River Laboratories). Cre-responsive Rosa26TdTomato (R26-TdT) reporter mice ${ }^{54}$ were crossbred with Myh6-mER-Cre-mER mice 55 to generate Myh6-mER-Cre-mER R26-TdT mice in a B6SV129F1 background. Tamoxifen dissolved in corn oil was administered prior to myocardial infarction (20 $\mathrm{mg} / \mathrm{kg}$ ). All animal studies were performed in accordance with local institutional guidelines and regulations.

Production of recombinant AAV vectors. The precursors of mmu-miR-106b, mmumiR-93 and mmu-miR-25 plus upstream and downstream flanking region sequences 
(total approximately 200 base pairs) were amplified from mouse genomic DNA isolated from a wild-type CD1 mouse heart, using QIAamp DNA mini kit (Qiagen), according to the manufacturer's instructions. The primers used to amplify the precursor sequences were: foward primer: 5'-GTATCATAAGGATCCCTTTCCACTGCTCTGGTGAG-3' and reverse primer: 3'- GTATCATAAGTCGACCTCACCTAGCTGTCTGTCC-5'. The amplified sequences were cloned into the pZac2.1 vector (Gene Therapy Program, Penn Vector core, University of Pennsylvania, USA) using the restriction enzymes BamH I and Sal I. Recombinant AAV serotype 9 vectors were generated at the AAV Vector Unit of ICGEB, Trieste (Italy), as described previously. ${ }^{36}$ CD1 mice at postnatal day 1 were intraperitoneally injected with an empty AAV9 vector (AAV9-MCS; multiple cloning site, negative control) or AAV9-miR-106b 25 at a dose of $1 \times 10^{11}$ viral genome particles per animal, using an insulin syringe with 30-gauge needle. 12 days after injection, the hearts were collected for histological analysis.

\section{Aortic banding, myocardial infarction, AAV9 delivery and transthoracic}

echocardiography. Transverse aortic constriction (TAC) or sham surgery was performed in 2-6 month-old B6SV129F1 mice by subjecting the aorta to a defined 27 gauge constriction between the first and second truncus of the aortic arch as described previously. ${ }^{56,57}$ Myocardial infarction (MI) was produced in 2-6 month-old CD1 mice by permanent left anterior descending (LAD) coronary artery ligation. ${ }^{36}$ Briefly, mice were anesthetized with an intraperitoneally injection of ketamine and xylazine, endotracheally intubated and placed on a rodent ventilator. Body temperature was maintained at $37^{\circ} \mathrm{C}$ on a heating pad. The beating heart was accessed via a left thoracotomy. After removing the pericardium, a descending branch of the LAD coronary artery was visualized with a stereomicroscope (Leica) and occluded with a nylon suture. Ligation was confirmed by the whitening of a region of the left ventricle, immediately post-ligation. Immediately after MI surgery, adult mice received an intracardiac injection of AAV9 vectors (AAV9-MCS or AAV9-miR-106b 25) at a dose of $1 \times 10^{11}$ viral genome particles per animal. 5-ethynyl$2^{\prime}$-deoxyuridine (EdU, Life Technologies) was administered intraperitoneally (500 $\mu \mathrm{g}$ per animal) every 2 days, for a period of ten days. For Doppler-echocardiography, mice were shaved and lightly anaesthetized with isoflurane (mean $3 \%$ in oxygen) and allowed to breathe spontaneously via a nasal cone. Non-invasive, echocardiographic parameters were measured using a RMV707B (15-45 MHz) scan-head interfaced with a Vevo-770 
high frequency ultrasound system (VisualSonics). Long-axis ECG-triggered cine loops of the left ventricular (LV) contraction cycle were obtained in B-mode to assess enddiastolic/systolic volume. Short-axis recordings of the LV contraction cycle were taken in M-mode to assess wall thickness of the anterior/posterior wall at the mid-papillary level. Doppler was used to determine the ratio between early $(E)$ and late $(A)$ ventricular filling velocity (E/A ratio) and to calculate the pressure gradient between the proximal and distal sites of the transverse aortic constriction and only mice with a pressure gradient > $50 \mathrm{~mm} \mathrm{Hg}$ were included. From B-mode recordings, LV length from basis to apex, LV internal diameter in systole (LVIDs) and diastole (LVIDd) were determined. From Mmode recordings, LV posterior wall thickness in systole (LV PWs) and diastole (LV PWd) were determined. LV mass was calculated with the following formula:

$\left(0,8^{*}\left(1.04^{*}\left(\left((\text { LVIDd + LV PWd + IVSd })^{\wedge} 3\right)-\left((\text { LVIDd })^{\wedge} 3\right)\right)+0,6\right) ;\right.$ fractional shortening (FS) was calculated with the following formula: (LVIDd-LVIDs)/LVIDd*100). Ejection fraction $(E F)$ was calculated as $\left((\mathrm{SV} / \mathrm{Vd})^{\star} 100\right)$ with $\mathrm{Vs}$, systolic volume $\left(3,1416^{*}\left(\mathrm{LVIDs}^{\wedge} 3\right) / 6\right), \mathrm{Vd}$, diastolic volume $\left(3,1416^{\star}\left(\operatorname{LVIDd}^{\wedge} 3\right) / 6\right)$, and SV, stroke volume $(\mathrm{Vd}-\mathrm{Vs}) . .^{57}$

Fluorescent Fluorescence activated cell sorting (FACS). Neonatal CD1 mice at age p1 randomly received AAV9-MCS or AAV9-miR106b 25. After 10 days, all mice we administered a single EdU injection and 2 days later cardiomyocytes were isolated and fixed using 4\% PFA. Next, cells were permeabilized using $0.1 \%$ Triton- $X$ and incubated for 2 hours at room temperature with mouse monoclonal antibody against sarcomeric $a-$ actinin (1:100; Abcam), followed by an incubation of 1 hour with the secondary antibody conjugated to Alexa Fluor-488 (Life Technologies). Next, cells were further processed using the Click-IT EdU 647 Imaging kit to reveal EdU incorporation, according to the manufacturer's instructions, and stained with Hoechst 33342 (Life Technologies). Acquisition and analysis was performed on a BD FACSCelesta cell analyser. Analysis was performed using FACSDiva Version 6.1.3.

Histological analysis and (immunofluorescence) microscopy. Hearts were arrested in diastole, perfusion fixed with $4 \%$ paraformaldehyde/PBS solution, embedded in paraffin and sectioned at $4 \mu \mathrm{m}$. Paraffin sections were stained with hematoxylin and eosine (H\&E) for routine histological analysis; Sirius Red for the detection of fibrillar collagen; and FITC-labelled antibody against wheat-germ-agglutinin (WGA) to visualize 
and quantify the myocyte cross-sectional area (1:100 Sigma Aldrich). Cell surface areas and Fibrotic areas were determined using ImageJ imaging software (http://rsb.info.nih.gov/ij/). For immunofluorescence, paraffin sections were deparaffinized, rehydrated, and permeabilized with $0.5 \%$ Triton X-100/PBS, followed by overnight incubation at $4{ }^{\circ} \mathrm{C}$ in $1 \% \mathrm{BSA}$ with primary antibodies: mouse monoclonal [EA53] to sarcomeric alpha-actinin (Abcam ab9465), rabbit polyclonal anti-phospho-Histone H3 (Ser10) (Sigma-Millipore 06-570), rabbit polyclonal anti-Aurora B (Abcam ab2254). Next, sections were washed with PBS and incubated for with the respective secondary antibodies conjugated with Alexa Fluor-488, -555, or -647. For Edu staining sections were processed with the Click-IT EdU 555 Imaging kit to according the manufacturer's instructions. The nuclear counter-staining was performed with Hoechst 33342 (Life Technologies) and slides were then mounted in Vectashield (Vector Labs). Slides were visualized using a Zeiss Axioskop 2Plus with an AxioCamHRc.

Quantification of cardiomyocytes and binucleation. Procedures were described in detail previously. ${ }^{24,26}$ Hearts were injected with $4 \mathrm{M} \mathrm{KCl}$ in the LV for cardioplegia, cut into 1-2 mm pieces and transferred to a peel-a-way embedding mold containing prewarmed $8 \%$ gelatin in DPBS at $37^{\circ} \mathrm{C}$. Tissue was frozen using dry-ice chilled isopentane and stored at $-80^{\circ} \mathrm{C}$. Forty $\mu \mathrm{m}$ sections were prepared using a cryostat and placed on positively charged glass slides, allowed to dry for $30 \mathrm{~min}$ at room temperature (RT). Cryosections were incubated for $40 \mathrm{~min}$ in pre-heated DPBS at $37^{\circ} \mathrm{C}$ to remove the gelatin, fixed using $4 \%$ formaldehyde in DPBS for 15 min at RT, washed twice in $3 \%$ bovine serum albumin (BSA) in DPBS for 5 min and then permeabilized with $1 \%$ Triton X-100 for 25 min at RT. Sections were incubated with EdU reaction buffer and washed 5 times in DPBS for 3 min. Blocking was done by incubating the tissue with $4 \%$ fetal bovine serum (FBS) in 1\% BSA in DPBS for $1 \mathrm{~h}$ at $\mathrm{RT}$, prior to incubation with primary antibody (pericentriolar material 1 (PCM-1) rabbit lgG, $0.4 \mathrm{mg} / \mu \mathrm{l}$ in blocking solution) overnight, at $4^{\circ} \mathrm{C}$, followed by incubation with secondary antibody (goat anti-rabbit 488 , $4 \mathrm{mg} / \mu \mathrm{l}$ in blocking solution) for $3 \mathrm{~h}$. Cell membranes were stained and nuclei counterstained by incubating the sections with wheat germ agglutinin (WGA) $(50 \mu \mathrm{g} / \mathrm{ml})$ and 4',6'-diamino-2-fenil-indol (DAPI) $(10 \mu \mathrm{g} / \mathrm{ml})$ for $1 \mathrm{~h}$ at $\mathrm{RT}$, respectively, and mounted in fluoroshield mounting media without DAPI and slides sealed using nail polish. Images of LV fragments were produced in a Leica SP8 STED laser scanning confocal 
microscope. PCM-1 signal was used to define the beginning and ending of Z-Stack, which varied depending on the penetrance of the PCM-1 antibody. Images were composed of an average number of 40-50 stacks, corresponding to a thickness of 20-25 $\mu \mathrm{m}$. Five images, from different fragments, were collected per heart. After image acquisition, images were quantified with assistance of FIJI and IMARIS. PCM-1+, EdU+ cells were considered when there was an overlap between PCM-1 (green), EdU (red) and DAPI (blue). Cardiomyocyte density and binucleation were determined for each individual heart by averaging the result obtained in the different fragments. The average number of nuclei per cardiomyocyte was calculated using the formula: average CM nuclei number $=2 \times(\%$ binucleation $)+1 \times(\%$ mononucleation $)$.

Western blot analysis. Whole tissue or cell lysates were produced in $150 \mathrm{mM} \mathrm{NaCl}, 50$ mM Tris- $\mathrm{HCl}, 5 \mathrm{mM}$ EDTA, $50 \mathrm{mM} \mathrm{NaF,} \mathrm{1 \%}$ Igepal $^{\mathrm{TM}}$, 0,05 \% SDS, $40 \mathrm{mM} \beta$ glycerophosphate, $10 \mathrm{mM}$ Na-pyrophosphate, PhosSTOP- and Protease inhibitor cocktail (Roche Applied Science). Samples were boiled in 4x Leammli buffer, including $2 \% \beta$-mercaptoethanol, for 5 minutes at $95^{\circ} \mathrm{C}$. SDS-PAGE and Western blotting were performed using the Mini-PROTEAN 3 system (Biorad). Blotted membranes were blocked in 5\% BSA / TBS-Tween. Primary antibody labeling was performed overnight at $4^{\circ} \mathrm{C}$ at a concentration of $2 \mu \mathrm{g} \operatorname{lgG}$ per $7 \mathrm{~mL}$ blocking buffer. Antibodies used included were: rabbit polyclonal antibody anti-p57 (H-91) (SantaCruz sc-8298), rabbit polyclonal antibody anti-E2f5 (Abcam ab22855), rabbit polyclonal antibody anti-E2F5 (E-19) (SantaCruz sc-999), rabbit monoclonal antibody anti-Cyclin E1 (D7T3U) (Cell Signaling Technology \#20808), rabbit polyclonal antibody anti-Wee1 (Cell Signaling Technology \#4936), rabbit polyclonal antibody anti-Mef2d (Abcam ab104515), mouse monoclonal anti-GAPDH (Millipore, MAB374 clone 6C5), mouse monoclonal anti-alpha-Tubulin (Sigma-Aldrich T6074) rabbit polyclonal anti-Histone H3 (Cell Signaling Technology 9715S) and the secondary polyclonal swine anti-rabbit immunoglobulins/HRP (DAKO P0399) and polyclonal rabbit anti-mouse immunoglobulins/HRP (DAKO P0161). Secondary HRP conjugated antibodies were applied for 1 hour at room temperature. Following antibody incubation, blots were washed for $3 \times 10$ minutes in TBS-Tween. Images were generated using Supersignal West Dura Extended Duration ECL Substrate (Pierce) and the LAS-3000 documentation system (FujiFilm Life Science). Stripping was performed with Restore Western blot stripping buffer (Pierce). Output intensities were 
normalized for loading.

Quantitative PCR. Total RNA (1 $\mu \mathrm{g})$ was was extracted using miRNeasy Mini Kit (Qiagen) and applied to either miR-based or mRNA based reverse transcription. For miR-based reverse transcription, total RNA was reverse transcribed using miRCURY LNA Universal cDNA synthesis kit (Exiqon) followed by Real-time PCR using predesigned miRCURY LNA PCR primer sets (Exiqon) and miRCURY LNA SYBR Green master mix, according to the manufacturer's instructions. ${ }^{57}$ Expression was normalized to expression levels of 5S rRNA. For mRNA-based reverse transcription, total RNA was reverse transcribed using hexameric random primers. The housekeeping gene ribosomal protein $L 7$ (RPL7) was used for normalization. Fold changes were determined using the $2^{-\triangle \Delta C T}$ method. Real-time PCR primer sequences used in the study are: mouse Nppa, 5'-TCTTCCTCGTCTTGGCCTTT-3' and 3'-

CCAGGTGGTCTAGCAGGTTC-5'; mouse Nppb, 5'-TGGGAGGTCACTCCTATCCT-3' and 3'-GGCCATTTCCTCCGACTTT-5'; mouse Acta1, 5'-

CCGGGAGAAGATGACTCAAA-3' and 3'-GTAGTACGGCCGGAAGCATA-5'; mouse Myh7, 5'-CGGACCTTGGAAGACCAGAT-3' and 3'-GACAGCTCCCCATTCTCTGT-5'; mouse Rpl7, 5'-GAAGCTCATCTATGAGAAGGC-3' and 3'AAGACGAAGGAGCTGCAGAAC-5'.

Primary cardiomyocyte cultures and transfections. Cardiomyocyte cultures were isolated by enzymatic dissociation of 1 day-old neonatal rat hearts and processed for immunofluorescence as described previously. ${ }^{58}$ Neonatal cardiomyocytes were seeded on Primaria 384-well plates (for microscopy) or in Primaria $10 \mathrm{~cm}$ dishes (for western blotting) and one day later, cells were transfected with mimics (Life Technologies) of hsa-miR-106b-5p, hsa-miR-93-5p, hsa-miR-25-3p or cel-miR-67 as control (25mM) using (Lipofectamine RNAiMAX, Life Technologies). Twenty-four hours after transfection, culture medium was replaced by fresh medium; and $52 \mathrm{~h}$ after plating $5 \mu \mathrm{M}$ 5-ethynyl-2'-deoxyuridine (EdU, Life Technologies) was added for $20 \mathrm{~h}$. For siRNA transfection, selected siRNAs (Dharmacon) at a final concentration of $50 \mathrm{nM}$ were transfected in cardiomyocytes seeded at $7.5 \times 10^{3}$ cells per well in collagen-coated black 
clear-bottom 384-well plates (PerkinEImer). ${ }^{36}$ Cells were fixed at $72 \mathrm{~h}$ after plating and processed for immunofluorescence.

Immunofluorescence and image acquisition of cardiomyocytes. Cells were fixed with $4 \%$ paraformaldehyde for $15 \mathrm{~min}$, permeabilized with $0.5 \%$ Triton $\mathrm{X}-100$ in phosphate buffered saline (PBS) solution for $10 \mathrm{~min}$, followed by $30 \mathrm{~min}$ blocking in $1 \%$ BSA (Roche). Cells were stained overnight at $4{ }^{\circ} \mathrm{C}$ with mouse monoclonal antibody against sarcomeric a-actinin (1:100; Abcam), followed by a secondary antibody conjugated to Alexa Fluor-488 (Life Technologies). Next, cells were further processed using the Click-IT EdU 555 Imaging kit to reveal EdU incorporation, according to the manufacturer's instructions, and stained with Hoechst 33342 (Life Technologies). Image acquisition was performed using an ImageXpress Micro automated high-content screening fluorescence microscope at 10x magnification; a total of 16 images were acquired per wavelength, well and replicate, corresponding to approximately 2.500 cells analyzed per condition. Image analysis was performed using the 'Multi-Wavelenght Cell Scoring' application module implemented in MetaXpress software (Molecular Devices). ${ }^{36}$ Proliferating cardiomyocytes were identified by a positive signal for the proliferation marker EdU and a positive signal for sarcomeric a-actinin.

Luciferase-reporter assays. Constructs bearing 309 bp of murine E2f5 3'UTR (pMIRE2f5), 591 bp of murine Cdkn1a 3'UTR (pMIR- Cdkn1a), 625 bp of murine Cdkn1c 3'UTR (pMIR- Cdkn1c) and 656 bp of murine Mef2d 3'UTR (pMIR- Mef2d) were subcloned into the pmirGLO Dual-Luciferase miRNA Target Expression Vector (Promega). Correspondent seed-sequence mutated Dual-pMIR-report plasmids were obtained using the QuikChange XL Site-Directed Mutagenesis Kit (Agilent Technologies). Low-passage COS7 cells were grown in DMEM (Invitrogen) supplemented with $10 \%$ FCS and seeded $\left(2,5 \times 10^{4}\right)$ in 48-well plates and transfected at $50-60 \%$ confluence with a total of 100 ng Dual-pMIR-report plasmids using XtremeGENE 9 DNA Transfection Reagent (Roche), followed by transfection with mimics (Life Technologies) of hsa-miR-106b-5p, hsa-miR-93-5p, hsa-miR-25-3p or cel-miR-67 as control (25mM) using Oligofectamine (Invitrogen). Firefly and Renilla luciferase activities were measured using the Dual Luciferase Reporter Assay System (Promega), according to the manufacturer's instructions. 


\section{Transcriptomic analysis and clustering of fold change expression. Deep-} sequencing of total RNA isolated from neonatal rat cardiomyocyte cultures was performed $72 \mathrm{hr}$ after transfection of mimics (Life Technologies) of hsa-miR-106b-5p, hsa-miR-93-5p, hsa-miR-25-3p or cel-miR-67 as control (25mM) by IGA Technology Services (Italy). ${ }^{36}$ RNA purity, integrity and concentration were determined using an Agilent 2100 Bioanalyzer (Agilent Technologies). Only RNAs with a RIN value $>7$ and an rRNA $28 S / 18 S$ ratio $>2$ were considered for sample preparation. Two $\mu \mathrm{g}$ of total RNA per sample was sequenced on an Illumina HiSeq2000. Two lanes in 7-plex were run obtaining 2 millions of single-reads per sample, 50-bp long. Real-time image analysis, base calling, de-multiplexing and production of FASTQ sequence files were performed on the HiSeq2000 instrument using the HiSeq Software. Raw sequence files were quality checked using FASTQC software (www.bioinformatics.babraham.ac.uk/projects/fastqc) and trimmed to remove Illumina adaptor using Cutadapt software. The raw sequencing reads were then mapped to Ensembl Rattus norvegicus reference genome (GCA_000001895.4 Rnor 6.0.89.6) ${ }^{59}$ using STAR software. Rounded gene counts were normalized to RPKM (reads per kilobase of exon model per million mapped reads) using the rpkm function in the Bioconductor package edgeR. ${ }^{60}$ Genes with RPKM values greater than 2.00 in both miRNA and cel-miR-67 transfected rat CMs were considered as expressed genes. Fold changes were taken with respect to the expression upon cel-miR-67transfection. Genes whose expression fold change were greater than 1.3 were considered as differentially expressed.

\section{Bioinformatics analyses: clustering of fold change expression; pathway} enrichment analysis; target predictions; network analyses. The Pearson correlation between the log2-fold changes for all pairs of miRNA were calculated. Clustering was performed hierarchically using the average linkage criterion with a Euclidean distance metric as implemented in SciPy v0.18.1 (http://www.scipy.org). A dendrogram was then generated using the SciPy clustering package to visualize the arrangement of the resulting cluster. Statistically enriched pathways from the set of differentially expressed genes were determined using a hypergeometric distribution-based statistical method as implemented in the Database for Annotation, Visualization and Integrated Discovery 
(DAVID) Bioinformatics Resources 6.8. The calculated $P$-values were then corrected according to Benjamini-Hochberg procedure to control the false discovery rates arising from multiple testing. KEGG pathways with $P<0.01$ and Benjamini-Hochberg FDR $<0.05$ are considered as statistically significant. Since bioinformatic predictions of seed sequence interactions with rat transcripts are not available, we compiled a list of rat miRNA-gene interactions from mouse predictions. Predicted mouse gene targets of the seed sequences (corresponding to miRNA families) of miRNAs which belong to the miR106b 25 cluster were collected from TargetScanMouse Release 7.1. The scores were calculated to be the most efficient interaction between a mouse gene and a human miRNA in a given miRNA family as determined by the seed sequence. The mouse genes were then translated to its corresponding rat genes through homology using the HomoloGene database. The list of miRNA-gene interactions was filtered to only include genes that were downregulated by the miRNA upon transfection to CMs according to the transcriptomic data (397 downregulated bioinformatic targets by miR-106b, 429 by miR93 and 358 by miR-25). A bioinformatic protein-protein interaction network was derived from STRING database v10.5, which integrates and scores protein interactions across different evidence channels (conserved neighborhood, co-occurrence, fusion, coexpression, experiments, databases and text mining) and combines the scores from these channels. Only those interactions solely involving rat proteins were considered. To obtain a high confidence interaction network, a cut-off score of 700 (out of 1000) on the combined score was imposed. For each miRNA, a subnetwork was extracted containing only the differentially expressed genes upon imposing a 1.3 fold-change cut-off and the genes of interest in this study. These subnetworks were then merged into a single multinetwork wherein the gene components can now be connected by three interactions, one for each miRNA. To further elucidate the multi-network, we catalogued the set of genes that are involved to biological processes of interest using the EMBL annotations of gene ontologies. These biological processes include cell division (G0:0051301 and GO:0000086) with 212 annotated genes, actin cytoskeleton (GO:0015629, GO:0030036, GO:0031532 and GO:0008154) with 345 annotated genes and oxidative stress response (GO:0006979) with 143 annotated genes. Furthermore, knowing the importance of the Hippo signaling pathway in cardiomyocyte proliferation, we also catalogued the set of genes involved in the canonical ${ }^{61}$ (19 genes) and non-canonical ${ }^{62}$ ( 21 genes) pathways in which Yap can be phosphorylated. These gene sets were then used to extract a 
compartmentalized network of genes that are differentially expressed after transfection of the miR-106b 25 cluster whose interactions can lead to cytokinesis. Network analysis was done using the NetworkX v1.11 package in Python while graph visualization was done using Cytoscape v3.4.0.

Statistical analysis. The results are presented as mean \pm standard error of the mean (SEM). Statistical approaches for bioinformatics analyses are described above. All other statistical analyses were performed using Prism software (GraphPad Software Inc.), and consisted of One-way ANOVA or Two-way ANOVA followed by Tukey Multiple Comparison Test when group differences were detected at the $5 \%$ significance level, or Student's $t$-test when comparing two experimental groups. Differences were considered significant when $P<0.05$.

Acknowledgements: E.D. is supported by a VENI award 916-150-16 from the Netherlands Organization for Health Research and Development (ZonMW), an EMBO Long-term Fellowship (EMBO ALTF 848-2013) and a FP7 Marie Curie Intra-European Fellowship (Project number 627539). P.D.C.M. is an Established Investigator of the Dutch Heart Foundation. L.D.W. acknowledges support from the Netherlands CardioVascular Research Initiative (CVON-ARENA-PRIME). L.D.W. was further supported by grant 311549 from the European Research Council (ERC), a VICI award 918-156-47 from the Dutch Research Council and Marie Sklodowska-Curie grant agreement no. 813716 (TRAIN-HEART).

Author Contributions: E.D., A.R., S.O., L.O. performed real time PCR experiments. A.R., H.A., S.O. performed Western blots. A.R. performed luciferase assays. E.D., C.T. and R.C. performed transcriptome analysis. H.A. and S.Z. performed surgical procedures in mouse models. E.D. performed echocardiography. E.D., A.R., V.S.P., D.S.W. performed histology in mouse models. E.D., A.R. analysed data. E.D. and S.M. performed FACS analyses. L.B. performed microscopic imaging. M.H., R.W., L.Z., J.V.B. and M.G. provided reagents and models. E.D., M.G. S.S., P.D.C.M. and L.D.W. designed the study. E.D., and L.D.W. wrote the manuscript. E.D, P.D.C.M. and L.D.W. acquired funding for the study. E.D. and A.R. contributed equally as joint first authors.

\section{Competing financial interests:}

E.D., M.G. and L.D.W filed the data in the manuscript for patent protection. P.D.C.M. and L.D.W. are co-founders and stockholders of Mirabilis Therapeutics BV.

Author Information: Correspondence and requests for materials should be addressed to L.D.W. (l.dewindt@maastrichtuniversity.nl). 


\section{REFERENCES}

1 Sedmera, D. \& Thompson, R. P. Myocyte proliferation in the developing heart. Dev Dyn 240, 1322-1334, doi:10.1002/dvdy.22650 (2011).

2 Soufan, A. T. et al. Three-dimensional measurement and visualization of morphogenesis applied to cardiac embryology. J Microsc 225, 269-274, doi:10.1111/j.1365-2818.2007.01742.x (2007).

3 Becker, R. O., Chapin, S. \& Sherry, R. Regeneration of the ventricular myocardium in amphibians. Nature 248, 145-147 (1974).

$4 \quad$ Flink, I. L. Cell cycle reentry of ventricular and atrial cardiomyocytes and cells within the epicardium following amputation of the ventricular apex in the axolotl, Amblystoma mexicanum: confocal microscopic immunofluorescent image analysis of bromodeoxyuridine-labeled nuclei. Anat Embryol (Berl) 205, 235-244, doi:10.1007/s00429-002-0249-6 (2002).

5 Gonzalez-Rosa, J. M., Martin, V., Peralta, M., Torres, M. \& Mercader, N. Extensive scar formation and regression during heart regeneration after cryoinjury in zebrafish. Development 138, 1663-1674, doi:10.1242/dev.060897 (2011).

6 Oberpriller, J. O. \& Oberpriller, J. C. Response of the adult newt ventricle to injury. J Exp Zool 187, 249-253, doi:10.1002/jez.1401870208 (1974).

7 Porrello, E. R. et al. Transient regenerative potential of the neonatal mouse heart. Science 331, 1078-1080, doi:10.1126/science.1200708 (2011).

8 Puente, B. N. et al. The oxygen-rich postnatal environment induces cardiomyocyte cell-cycle arrest through DNA damage response. Cell 157, 565579, doi:10.1016/j.cell.2014.03.032 (2014).

9 Heallen, T. et al. Hippo pathway inhibits Wnt signaling to restrain cardiomyocyte proliferation and heart size. Science 332, 458-461, doi:10.1126/science.1199010 (2011).

10 D'Uva, G. et al. ERBB2 triggers mammalian heart regeneration by promoting cardiomyocyte dedifferentiation and proliferation. Nat Cell Biol 17, 627-638, doi:10.1038/ncb3149 (2015).

11 Mahmoud, A. I. et al. Meis1 regulates postnatal cardiomyocyte cell cycle arrest. Nature 497, 249-253, doi:10.1038/nature12054 (2013).

12 Malek Mohammadi, M. et al. The transcription factor GATA4 promotes myocardial regeneration in neonatal mice. EMBO Mol Med 9, 265-279, doi:10.15252/emmm.201606602 (2017).

13 Aguirre, A. et al. In vivo activation of a conserved microRNA program induces mammalian heart regeneration. Cell Stem Cell 15, 589-604, doi:10.1016/j.stem.2014.10.003 (2014).

14 Tian, Y. et al. A microRNA-Hippo pathway that promotes cardiomyocyte proliferation and cardiac regeneration in mice. Sci Transl Med 7, 279ra238, doi:10.1126/scitranslmed.3010841 (2015).

15 Haubner, B. J., Schuetz, T. \& Penninger, J. M. A reproducible protocol for neonatal ischemic injury and cardiac regeneration in neonatal mice. Basic Res Cardiol 111, 64, doi:10.1007/s00395-016-0580-3 (2016).

16 Li, F., Wang, X., Capasso, J. M. \& Gerdes, A. M. Rapid transition of cardiac myocytes from hyperplasia to hypertrophy during postnatal development. J Mol Cell Cardiol 28, 1737-1746, doi:10.1006/jmcc.1996.0163 (1996). 
17 Bergmann, O. et al. Evidence for cardiomyocyte renewal in humans. Science 324, 98-102, doi:10.1126/science.1164680 (2009).

18 Maillet, M., van Berlo, J. H. \& Molkentin, J. D. Molecular basis of physiological heart growth: fundamental concepts and new players. Nat Rev Mol Cell Biol 14, 38-48, doi:10.1038/nrm3495 (2013).

19 Velazquez, E. J. et al. Coronary-Artery Bypass Surgery in Patients with Ischemic Cardiomyopathy. N Engl J Med 374, 1511-1520, doi:10.1056/NEJMoa1602001 (2016).

20 Dirkx, E. et al. Nfat and miR-25 cooperate to reactivate the transcription factor Hand2 in heart failure. Nat Cell Biol 15, 1282-1293, doi:10.1038/ncb2866 (2013).

21 Ventura, A. et al. Targeted deletion reveals essential and overlapping functions of the miR-17 through 92 family of miRNA clusters. Cell 132, 875-886, doi:10.1016/j.cell.2008.02.019 (2008).

22 Inagaki, K. et al. Robust systemic transduction with AAV9 vectors in mice: efficient global cardiac gene transfer superior to that of AAV8. Mol Ther 14, 4553, doi:10.1016/j.ymthe.2006.03.014 (2006).

23 Buja, L. M. et al. Characterization of a potentially reversible increase in betaadrenergic receptors in isolated, neonatal rat cardiac myocytes with impaired energy metabolism. Circ Res 57, 640-645 (1985).

24 Alkass, K. et al. No Evidence for Cardiomyocyte Number Expansion in Preadolescent Mice. Cell 163, 1026-1036, doi:10.1016/j.cell.2015.10.035 (2015).

Sampaio-Pinto, V. et al. Neonatal Apex Resection Triggers Cardiomyocyte Proliferation, Neovascularization and Functional Recovery Despite Local Fibrosis. Stem Cell Reports 10, 860-874, doi:10.1016/j.stemcr.2018.01.042 (2018).

26 Sampaio-Pinto, V. et al. Stereological estimation of cardiomyocyte number and proliferation. Methods, doi:10.1016/j.ymeth.2020.06.002 (2020).

27 Senyo, S. E. et al. Mammalian heart renewal by pre-existing cardiomyocytes. Nature 493, 433-436, doi:10.1038/nature11682 (2013).

28 Brodsky, V., Sarkisov, D. S., Arefyeva, A. M., Panova, N. W. \& Gvasava, I. G. Polyploidy in cardiac myocytes of normal and hypertrophic human hearts; range of values. Virchows Arch 424, 429-435 (1994).

29 Olson, E. N. \& Schneider, M. D. Sizing up the heart: development redux in disease. Genes Dev 17, 1937-1956, doi:10.1101/gad.1110103 (2003).

$30 \mathrm{Kim}, \mathrm{Y}$. et al. The MEF2D transcription factor mediates stress-dependent cardiac remodeling in mice. J Clin Invest 118, 124-132, doi:10.1172/JCI33255 (2008).

31 Busk, P. K. et al. Involvement of cyclin D activity in left ventricle hypertrophy in vivo and in vitro. Cardiovasc Res 56, 64-75 (2002).

32 Pasumarthi, K. B., Nakajima, H., Nakajima, H. O., Soonpaa, M. H. \& Field, L. J. Targeted expression of cyclin D2 results in cardiomyocyte DNA synthesis and infarct regression in transgenic mice. Circ Res 96, 110-118, doi:10.1161/01.RES.0000152326.91223.4F (2005).

33 Chaudhry, H. W. et al. Cyclin A2 mediates cardiomyocyte mitosis in the postmitotic myocardium. J Biol Chem 279, 35858-35866, doi:10.1074/jbc.M404975200 (2004).

34 Cheng, R. K. et al. Cyclin A2 induces cardiac regeneration after myocardial infarction and prevents heart failure. Circ Res 100, 1741-1748, doi:10.1161/CIRCRESAHA.107.153544 (2007). 
35 Shapiro, S. D. et al. Cyclin A2 induces cardiac regeneration after myocardial infarction through cytokinesis of adult cardiomyocytes. Sci Transl Med 6, 224ra227, doi:10.1126/scitranslmed.3007668 (2014).

36 Eulalio, A. et al. Functional screening identifies miRNAs inducing cardiac regeneration. Nature 492, 376-381, doi:10.1038/nature11739 (2012).

37 Wang, B. et al. miR-25 Promotes Cardiomyocyte Proliferation by Targeting FBXW7. Mol Ther Nucleic Acids 19, 1299-1308, doi:10.1016/j.omtn.2020.01.013 (2020).

38 Heallen, T. et al. Hippo signaling impedes adult heart regeneration. Development 140, 4683-4690, doi:10.1242/dev.102798 (2013).

39 von Gise, A. et al. YAP1, the nuclear target of Hippo signaling, stimulates heart growth through cardiomyocyte proliferation but not hypertrophy. Proc Natl Acad Sci U S A 109, 2394-2399, doi:10.1073/pnas.1116136109 (2012).

40 Xin, M. et al. Hippo pathway effector Yap promotes cardiac regeneration. Proc Natl Acad Sci U S A 110, 13839-13844, doi:10.1073/pnas.1313192110 (2013).

41 Xin, M. et al. Regulation of insulin-like growth factor signaling by Yap governs cardiomyocyte proliferation and embryonic heart size. Sci Signal 4, ra70, doi:10.1126/scisignal.2002278 (2011).

42 Leach, J. P. et al. Hippo pathway deficiency reverses systolic heart failure after infarction. Nature 550, 260-264, doi:10.1038/nature24045 (2017).

43 Ikeda, S. et al. Hippo Deficiency Leads to Cardiac Dysfunction Accompanied by Cardiomyocyte Dedifferentiation During Pressure Overload. Circ Res 124, 292305, doi:10.1161/CIRCRESAHA.118.314048 (2019).

44 Wahlquist, C. et al. Inhibition of miR-25 improves cardiac contractility in the failing heart. Nature 508, 531-535, doi:10.1038/nature13073 (2014).

45 Pan, L. et al. MiR-25 protects cardiomyocytes against oxidative damage by targeting the mitochondrial calcium uniporter. Int J Mol Sci 16, 5420-5433, doi:10.3390/ijms16035420 (2015).

46 Yao, Y., Sun, F. \& Lei, M. miR-25 inhibits sepsis-induced cardiomyocyte apoptosis by targetting PTEN. Biosci Rep 38, doi:10.1042/BSR20171511 (2018).

$47 \mathrm{Li}, \mathrm{H}$. et al. Alteration in microRNA-25 expression regulate cardiac function via renin secretion. Exp Cell Res 365, 119-128, doi:10.1016/j.yexcr.2018.02.029 (2018).

48 Chiang, D. Y. et al. Loss of microRNA-106b-25 cluster promotes atrial fibrillation by enhancing ryanodine receptor type-2 expression and calcium release. Circ Arrhythm Electrophysiol 7, 1214-1222, doi:10.1161/CIRCEP.114.001973 (2014).

49 Thum, T. et al. Comparison of different miR-21 inhibitor chemistries in a cardiac disease model. J Clin Invest 121, 461-462; author reply 462-463, doi:10.1172/JCl45938 (2011).

50 Gebert, L. F. et al. Miravirsen (SPC3649) can inhibit the biogenesis of miR-122. Nucleic Acids Res 42, 609-621, doi:10.1093/nar/gkt852 (2014).

51 Wang, J. et al. Pitx2-microRNA pathway that delimits sinoatrial node development and inhibits predisposition to atrial fibrillation. Proc Natl Acad Sci U $S$ A 111, 9181-9186, doi:10.1073/pnas.1405411111 (2014).

52 Gudbjartsson, D. F. et al. Variants conferring risk of atrial fibrillation on chromosome 4q25. Nature 448, 353-357, doi:10.1038/nature06007 (2007).

53 Molkentin, J. D. et al. A calcineurin-dependent transcriptional pathway for cardiac hypertrophy. Cell 93, 215-228 (1998). 
54 Chen, Z. \& van Berlo, J. H. Genetic Lineage Tracing of Non-cardiomyocytes in Mice. Methods Mol Biol 2158, 323-336, doi:10.1007/978-1-0716-0668-1_24 (2021).

55 Sohal, D. S. et al. Temporally regulated and tissue-specific gene manipulations in the adult and embryonic heart using a tamoxifen-inducible Cre protein. Circ Res 89, 20-25, doi:10.1161/hh1301.092687 (2001).

56 Armand, A. S. et al. Cooperative synergy between NFAT and MyoD regulates myogenin expression and myogenesis. J Biol Chem 283, 29004-29010, doi:10.1074/jbc.M801297200 (2008).

57 da Costa Martins, P. A. et al. MicroRNA-199b targets the nuclear kinase Dyrk1a in an auto-amplification loop promoting calcineurin/NFAT signalling. Nat Cell Biol 12, 1220-1227, doi:10.1038/ncb2126 (2010).

58 De Windt, L. J., Lim, H. W., Haq, S., Force, T. \& Molkentin, J. D. Calcineurin promotes protein kinase $\mathrm{C}$ and $\mathrm{c}$-Jun $\mathrm{NH}$-terminal kinase activation in the heart. Cross-talk between cardiac hypertrophic signaling pathways. J Biol Chem 275, 13571-13579 (2000).

59 Yates, A. et al. Ensembl 2016. Nucleic Acids Res 44, D710-716, doi:10.1093/nar/gkv1157 (2016).

60 McCarthy, D. J., Chen, Y. \& Smyth, G. K. Differential expression analysis of multifactor RNA-Seq experiments with respect to biological variation. Nucleic Acids Res 40, 4288-4297, doi:10.1093/nar/gks042 (2012).

61 Yu, F. X. \& Guan, K. L. The Hippo pathway: regulators and regulations. Genes Dev 27, 355-371, doi:10.1101/gad.210773.112 (2013).

62 Mohseni, M. et al. A genetic screen identifies an LKB1-MARK signalling axis controlling the Hippo-YAP pathway. Nat Cell Biol 16, 108-117, doi:10.1038/ncb2884 (2014). 
Table 1. Morphometric and echocardiographic characteristics of WT versus miR-106b 25 KO mice subjected to sham or TAC surgery for 4 weeks.

\begin{tabular}{|c|c|c|c|c|}
\hline & \multicolumn{2}{|c|}{ Sham } & \multicolumn{2}{|c|}{ TAC } \\
\hline & WT & KO & WT & KO \\
\hline $\mathbf{n}$ & 10 & 11 & 9 & 10 \\
\hline BW $(g)$ & $21.2 \pm 0.2$ & $21.3 \pm 0.4$ & $21.0 \pm 0.5$ & $21.0 \pm 0.4$ \\
\hline LV mass (mg) & $107 \pm 5$ & $80 \pm 5^{*}$ & $145 \pm 12^{*}$ & $110 \pm 8 \#$ \\
\hline LV mass/BW (mg/g) & $5.1 \pm 0.3$ & $3.8 \pm 0.4^{*}$ & $7.1 \pm 0.7 *$ & $5.3 \pm 0.5 \#$ \\
\hline IVSd $(\mathrm{mm})$ & $0.83 \pm 0.05$ & $0.68 \pm 0.03 *$ & $1.04 \pm 0.06^{*}$ & $0.89 \pm 0.04 \#$ \\
\hline IVSs (mm) & $1.16 \pm 0.06$ & $1.04 \pm 0.03$ & $1.40 \pm 0.07^{*}$ & $1.16 \pm 0.05 \#$ \\
\hline LVIDd (mm) & $4.09 \pm 0.08$ & $4.15 \pm 0.09$ & $4.10 \pm 0.14$ & $4.41 \pm 0.11$ \\
\hline LVIDs (mm) & $2.87 \pm 0.08$ & $3.14 \pm 0.08$ & $3.14 \pm 0.07$ & $3.61 \pm 0.15^{* \#}$ \\
\hline LVPWd (mm) & $0.89 \pm 0.04$ & $0.65 \pm 0.04 *$ & $1.04 \pm 0.06^{*}$ & $0.70 \pm 0.05 * \#$ \\
\hline LVPWs (mm) & $1.11 \pm 0.05$ & $0.83 \pm 0.03 *$ & $1.30 \pm 0.08^{*}$ & $0.88 \pm 0.05 * \#$ \\
\hline EF (\%) & $64 \pm 1$ & $57 \pm 1 *$ & $54 \pm 2 *$ & $45 \pm 3 * \#$ \\
\hline FS (\%) & $29 \pm 1$ & $25 \pm 1^{*}$ & $23 \pm 1 *$ & $18 \pm 1 * \#$ \\
\hline $\mathbf{E} / \mathbf{A}(\mathbf{m m} / \mathbf{s})$ & $1.73 \pm 0.11$ & $1.53 \pm 0.10$ & $1.62 \pm 0.10$ & $1.57 \pm 0.24 *$ \\
\hline
\end{tabular}

Data are expressed as means \pm SEM. BW, body weight; LV, left ventricular; IVSd, interventricular septal thickness at end-diastole; IVSs, interventricular septal thickness at endsystole; LVIDd, left ventricular internal dimension at end-diastole; LVIDs, left ventricular internal dimension at end-systole; LVPwd, left ventricular posterior wall thickness at enddiastole; LVPws, left ventricular posterior wall thickness at end-systole; EF, ejection fraction; FS, fractional shortening; E/A, Doppler E/A ratio. ${ }^{*}$, indicates $P<0.05$ vs sham group subjected to treatment with a control antagomir; \#, indicates $P<0.05$ vs experimental group. 
Table 2. Morphometric and echocardiographic characteristics of mice treated for 4 weeks with AAV9-MCS or AAV9-miR-106b 25.

\begin{tabular}{|r|cc|}
\cline { 2 - 3 } \multicolumn{1}{c|}{} & AAV9-MCS & AAV9-miR-106b 25 \\
$\mathbf{n}$ & 5 & 5 \\
BW (g) & $29.4 \pm 0.5$ & $24.3 \pm 1.7$ \\
LV mass (mg) & $79 \pm 7$ & $121 \pm 26^{*}$ \\
LV mass/BW (mg/g) & $2.7 \pm 0.2$ & $4.8 \pm 0.6^{*}$ \\
IVSd (mm) & $0.68 \pm 0.03$ & $0.91 \pm 0.08^{*}$ \\
IVSs (mm) & $1.05 \pm 0.04$ & $1.28 \pm 0.09$ \\
LVIDd (mm) & $3.75 \pm 0.12$ & $4.08 \pm 0.08^{*}$ \\
LVIDs (mm) & $2.50 \pm 0.14$ & $2.78 \pm 0.19$ \\
LVPWd (mm) & $0.84 \pm 0.09$ & $0.85 \pm 0.08$ \\
LVPWs (mm) & $1.07 \pm 0.06$ & $1.09 \pm 0.07$ \\
$\mathbf{E F ~ ( \% ) ~}$ & $70 \pm 3$ & $66 \pm 5$ \\
FS (\%) & $33 \pm 2$ & $31 \pm 3$ \\
\hline
\end{tabular}

Data are expressed as means \pm SEM. BW, body weight; LV, left ventricular; IVSd, interventricular septal thickness at end-diastole; IVSs, interventricular septal thickness at endsystole; LVIDd, left ventricular internal dimension at end-diastole; LVIDs, left ventricular internal dimension at end-systole; LVPwd, left ventricular posterior wall thickness at enddiastole; LVPws, left ventricular posterior wall thickness at end-systole; EF, ejection fraction; FS, fractional shortening; E/A, Doppler E/A ratio. *, indicates $P<0.05$ vs sham group subjected to treatment with a control antagomir; \#, indicates $P<0.05$ vs experimental group. 
Table 3. Morphometric and echocardiographic characteristics of mice subjected to sham or MI surgery and treated for 3 weeks with AAV9-MCS or AAV9-miR-106b 25.

\begin{tabular}{|c|c|c|c|c|}
\hline & \multicolumn{2}{|c|}{ Sham } & \multicolumn{2}{|c|}{ MI } \\
\hline & AAV9-MCS & $\begin{array}{l}\text { AAV9- } \\
\text { miR-106 25 }\end{array}$ & AAV9-MCS & $\begin{array}{l}\text { AAV9- } \\
\text { miR-106 25 }\end{array}$ \\
\hline $\mathbf{n}$ & 8 & 8 & 8 & 8 \\
\hline BW $(g)$ & $22.8 \pm 2.1$ & $25.3 \pm 2.0$ & $23.8 \pm 1.5$ & $24.3 \pm 1.7$ \\
\hline LV mass (mg) & $84 \pm 3$ & $90 \pm 2$ & $88 \pm 5$ & $107 \pm 4 * \#$ \\
\hline LV mass/BW (mg/g) & $3.2 \pm 0.2$ & $3.8 \pm 0.4$ & $5.2 \pm 0.4^{*}$ & $6.4 \pm 1.0^{*}$ \\
\hline IVSd (mm) & $0.79 \pm 0.03$ & $0.81 \pm 0.02$ & $0.75 \pm 0.03$ & $0.90 \pm 0.03 * \#$ \\
\hline IVSs (mm) & $1.18 \pm 0.05$ & $1.23 \pm 0.07$ & $1.10 \pm 0.04$ & $1.40 \pm 0.06^{*} \#$ \\
\hline LVIDd (mm) & $3.62 \pm 0.07$ & $3.78 \pm 0.10$ & $4.53 \pm 0.19^{*}$ & $4,00 \pm 0.02 \#$ \\
\hline LVIDs (mm) & $2.42 \pm 0.13$ & $2.56 \pm 0.14$ & $3.55 \pm 0.20^{*}$ & $2.79 \pm 0.26 \#$ \\
\hline LVPWd (mm) & $0.88 \pm 0.03$ & $0.86 \pm 0.05$ & $0.55 \pm 0.05^{*}$ & $0.87 \pm 0.10 \#$ \\
\hline LVPWs (mm) & $1.16 \pm 0.05$ & $1.21 \pm 0.06$ & $0.69 \pm 0.07^{*}$ & $1.05 \pm 0.13 \#$ \\
\hline EF (\%) & $69 \pm 4$ & $67 \pm 3$ & $52 \pm 2 *$ & $65 \pm 4 * \#$ \\
\hline FS (\%) & $33 \pm 3$ & $31 \pm 2$ & $22 \pm 1 *$ & $31 \pm 3 * \#$ \\
\hline
\end{tabular}

Data are expressed as means \pm SEM. BW, body weight; LV, left ventricular; IVSd, interventricular septal thickness at end-diastole; IVSs, interventricular septal thickness at endsystole; LVIDd, left ventricular internal dimension at end-diastole; LVIDs, left ventricular internal dimension at end-systole; LVPwd, left ventricular posterior wall thickness at enddiastole; LVPws, left ventricular posterior wall thickness at end-systole; EF, ejection fraction; FS, fractional shortening; E/A, Doppler E/A ratio. *, indicates $P<0.05$ vs sham group subjected to treatment with a control antagomir; \#, indicates $P<0.05$ vs experimental group. 
bioRxiv preprint doi: https://doi.org/10.1101/2021.02.25.432908; this version posted February 25, 2021. The copyright holder for this preprint (which was not certified by peer review) is the author/funder. All rights reserved. No reuse allowed without permission.

Figure 1

a

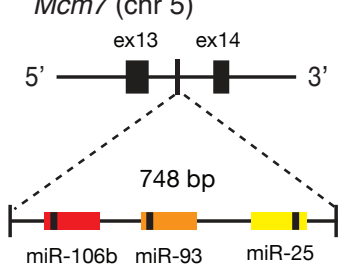

UAAAGUGCUGACAGUGCAGAU

CAAAGUGCUGUUCGUGCAGGUAG

I CAUUGCACUUGUCUCGguCUGa
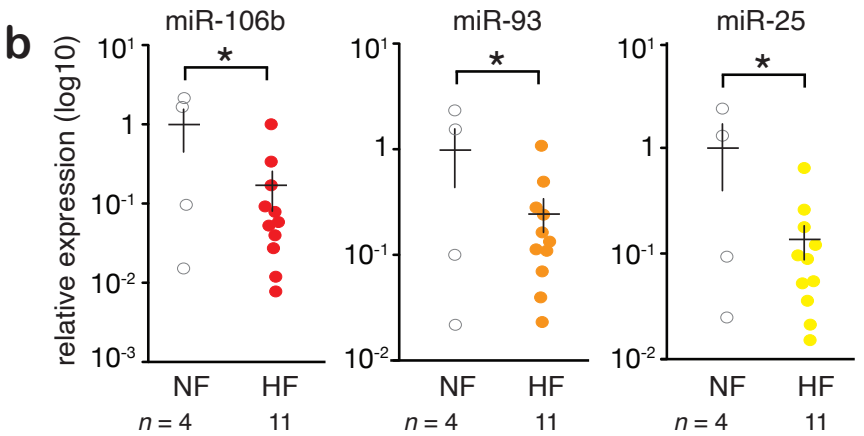

C

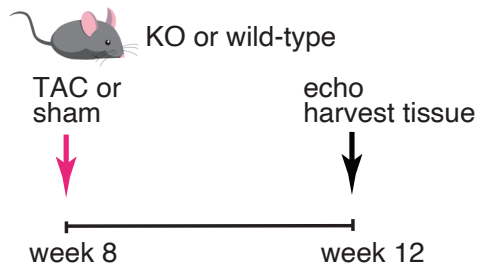

d

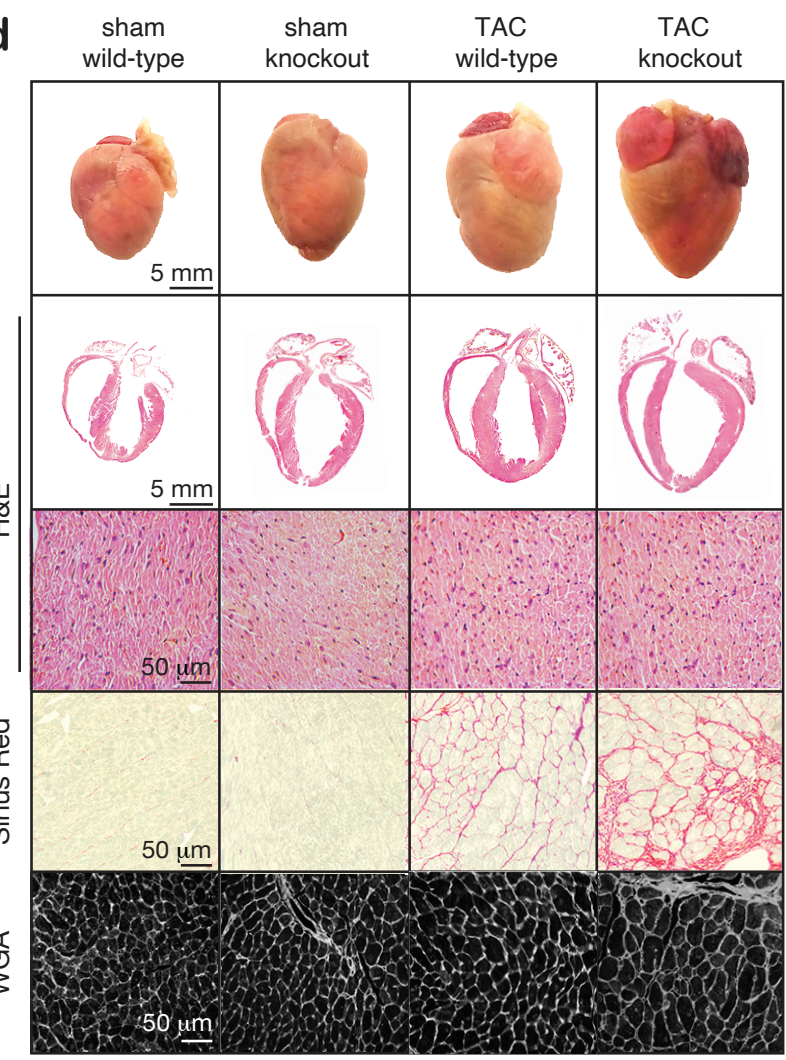

e

f
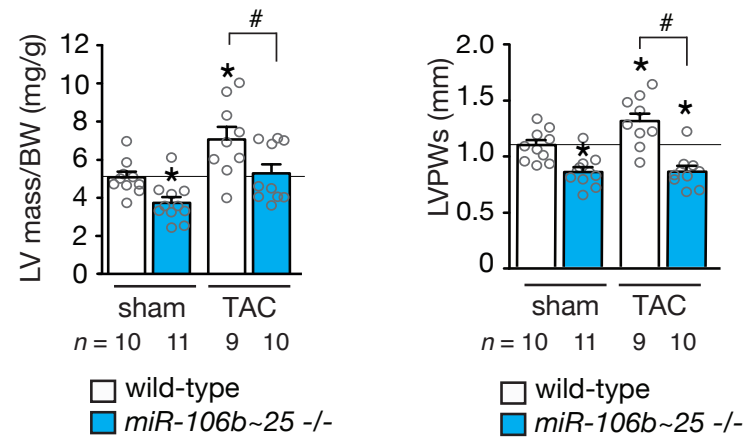

g

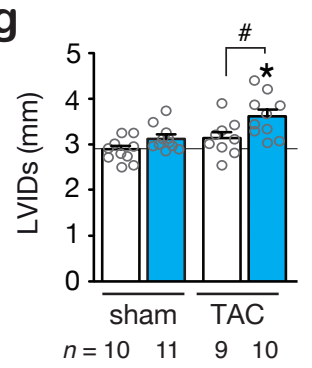

h

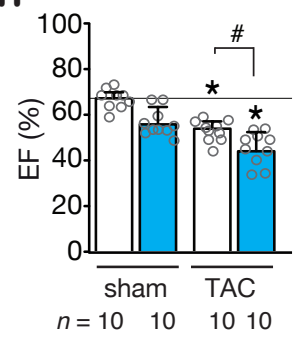

$\square$ wild-type

$\square$ miR-106b 25 -/-

$\square$ wild-type

$\square$ miR-106b 25 -/-

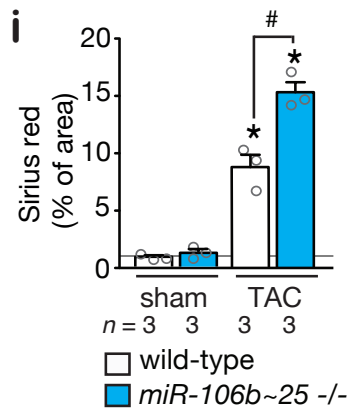

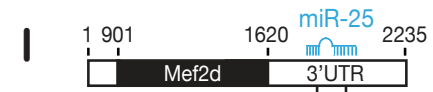

human $5, \quad \ldots \ldots . . . .$. hsa-miR-25 3' AǴŪC̄UGGCUCUGUUCACGŨÙ̇C 5' CUCUAAGAUAUACAGUGCAAUA 3' chimpansee 5' CUCUAAGAUAUACAGUGCAAUA 3' gorilla 5', CUCUAAGAUAUACAGUGCAAUA 3' horse 5' CUCUAAGAUAUACAGUGCAAUA 3' dog 5' CUCUAAGAUAUGCAGUGCAAUA 3' rabbit 5' CUCUAAGAUACACAGUGCAAUA 3' rat 5' CUUUAAGAUGUACAGUGCAAUA 3' mouse 5' CUUUGAGAUGUACAGUGCAAUA 3 '
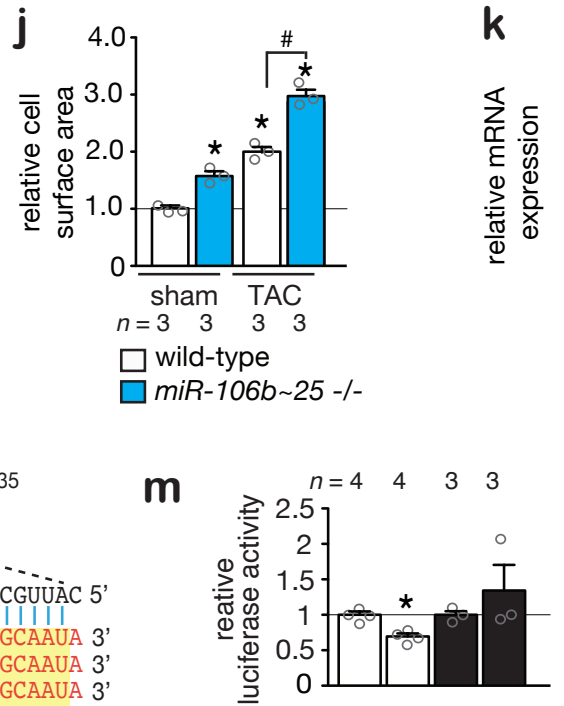

pmiR-Mef2d $+\quad+\quad-\quad-$ pmiR-Mef2d mut - -++ $\mathrm{scr}-\mathrm{miR}+-+-$ pre-miR-25 - + - + $\begin{array}{llll}\text { Nppa } & \text { Nppb } & \text { Acta1 } & \text { Myh7 }\end{array}$

k

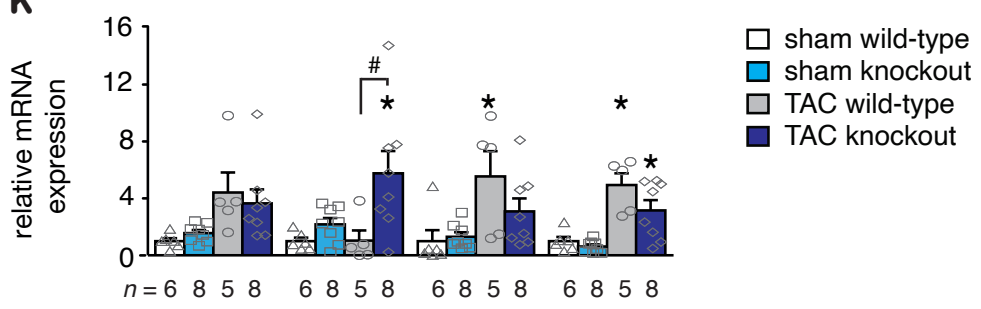

n

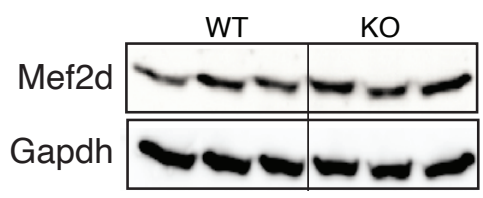

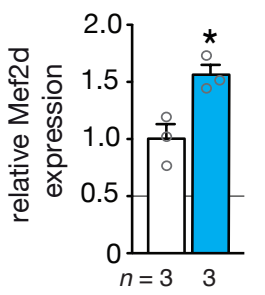

$\square$ wild-type

$\square$ miR-106b 25 -/- 
bioRxiv preprint doi: https://doi.org/10.1101/2021.02.25.432908; this version posted February 25, 2021. The copyright holder for this preprint (which was not certified by peer review) is the author/funder. All rights reserved. No reuse allowed without permission.

Figure 2

a

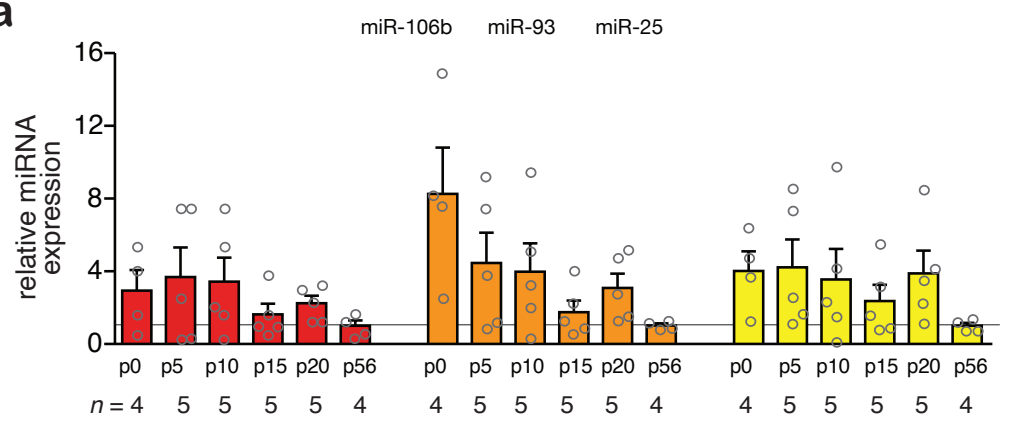

C
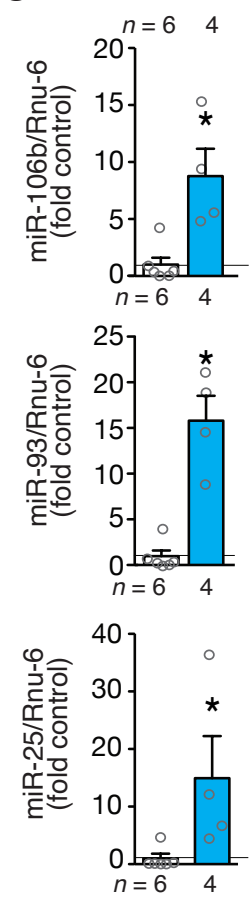

AAV9-MCS

AAV9-miR-106b 25
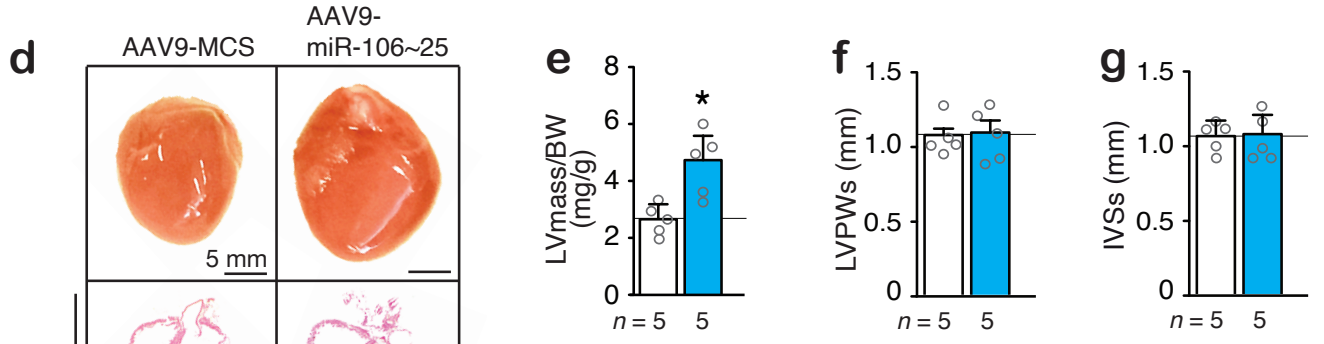

AAV9-MCS

$\square$ AAV9-miR-106b 25
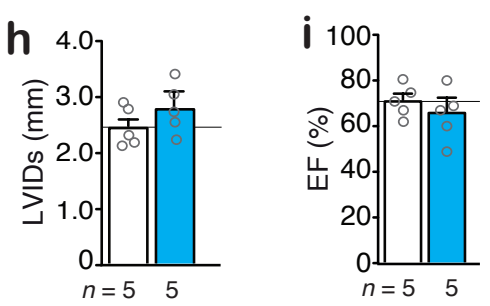

$\square$ AAV9-MCS

$\square$ AAV9-miR-106b 25 b

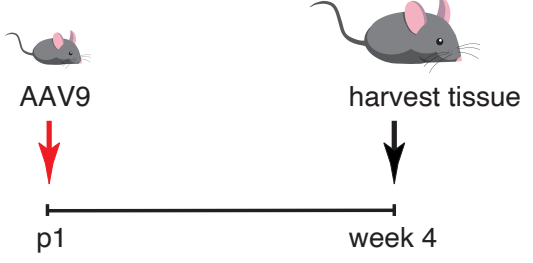

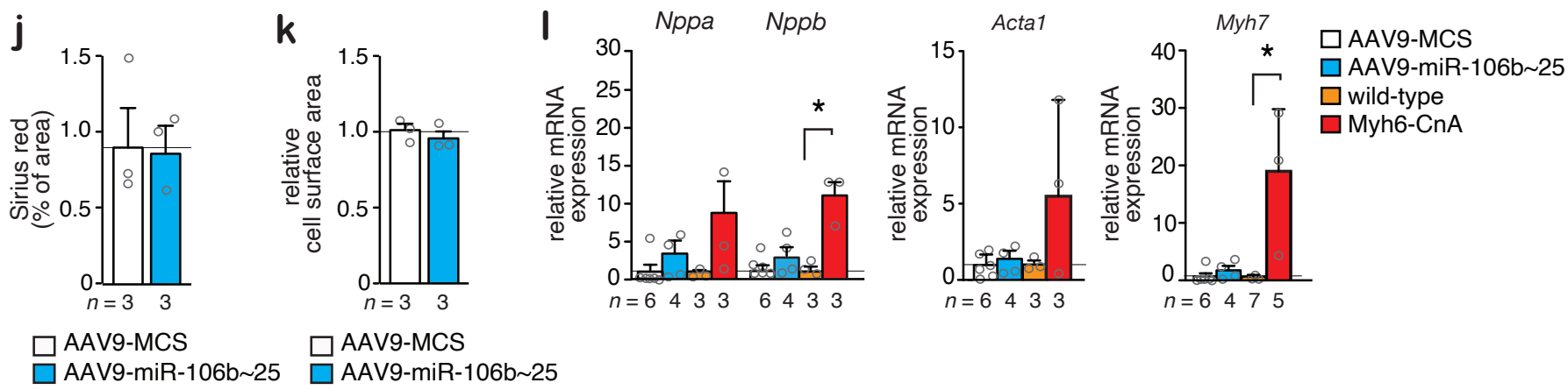


Figure 3

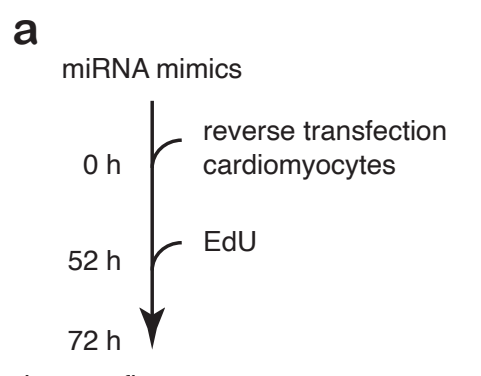

immunofluorescence image segmentation
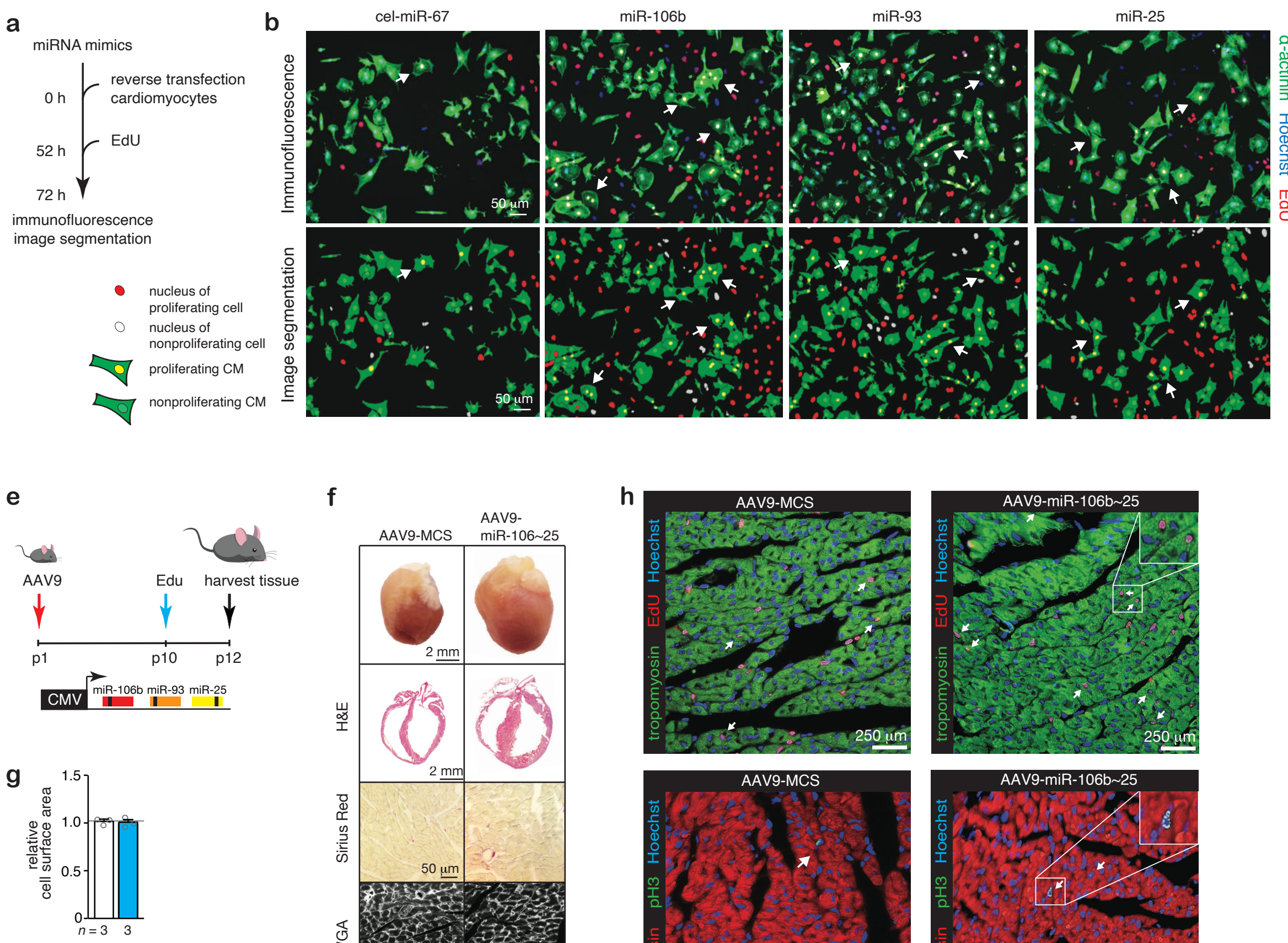

$\square$ AAV9-MCS

$\square$ AAV9-miR-106b 25

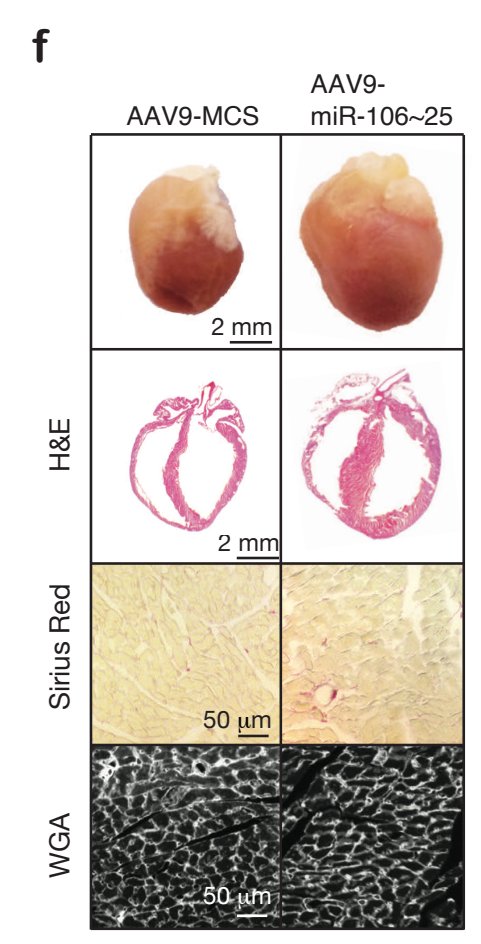

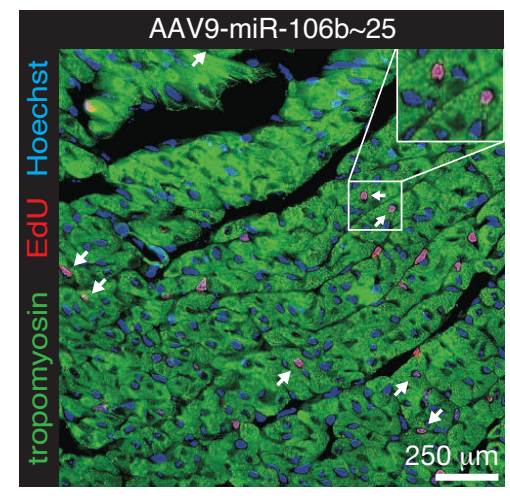

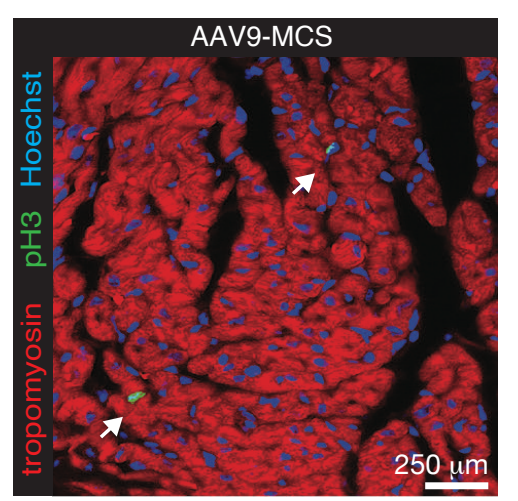

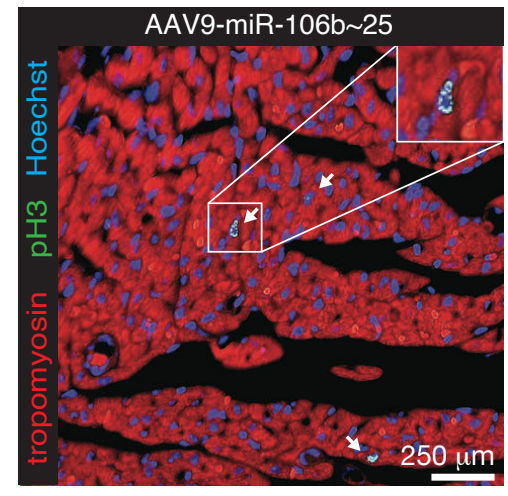

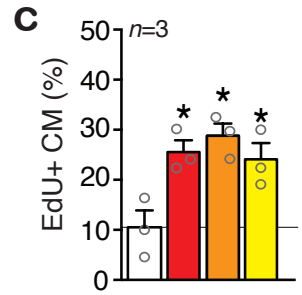

d $5000, n=3$ *
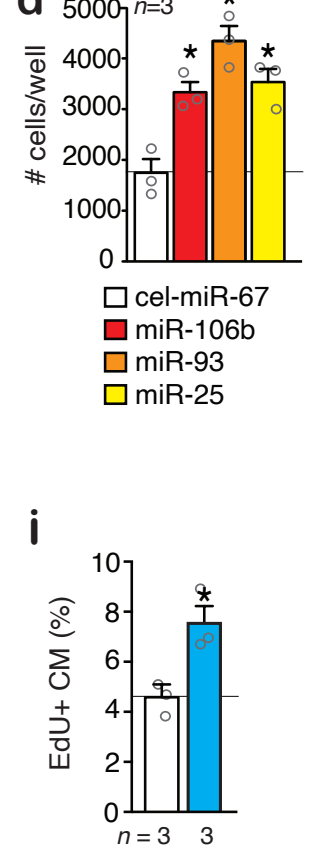

$\square$ AAV9-MCS

$\square$ AAV9-miR-106b 25

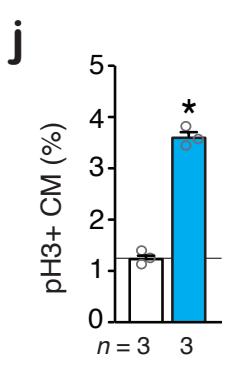

$\square$ AAV9-MCS

$\square$ AAV9-miR-106b 25 
bioRxiv preprint doi: https://doi.org/10.1101/2021.02.25.432908; this version posted February 25, 2021. The copyright holder for this preprint a (which was not certified by pefor review) is the author/funder. All rights reserved. No reuse allowed without permission.

KO or wild-type
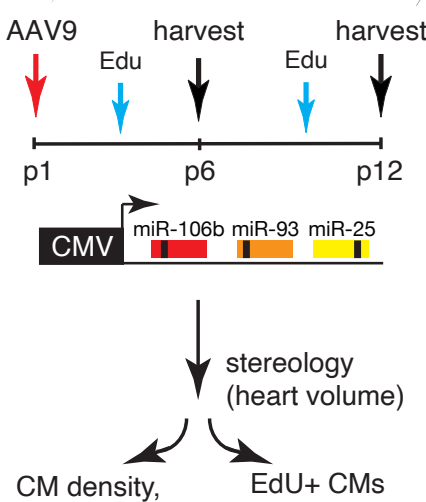

nucleation

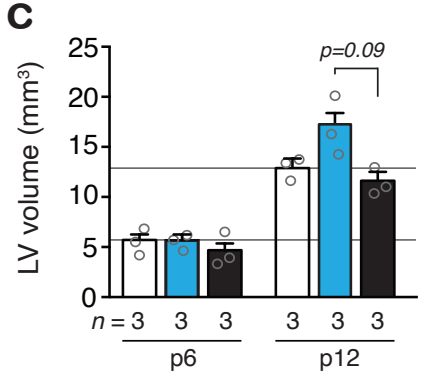

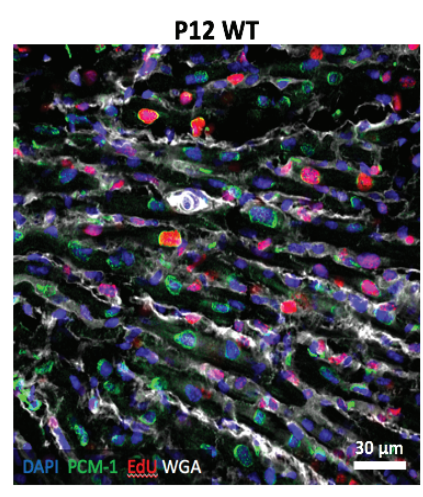
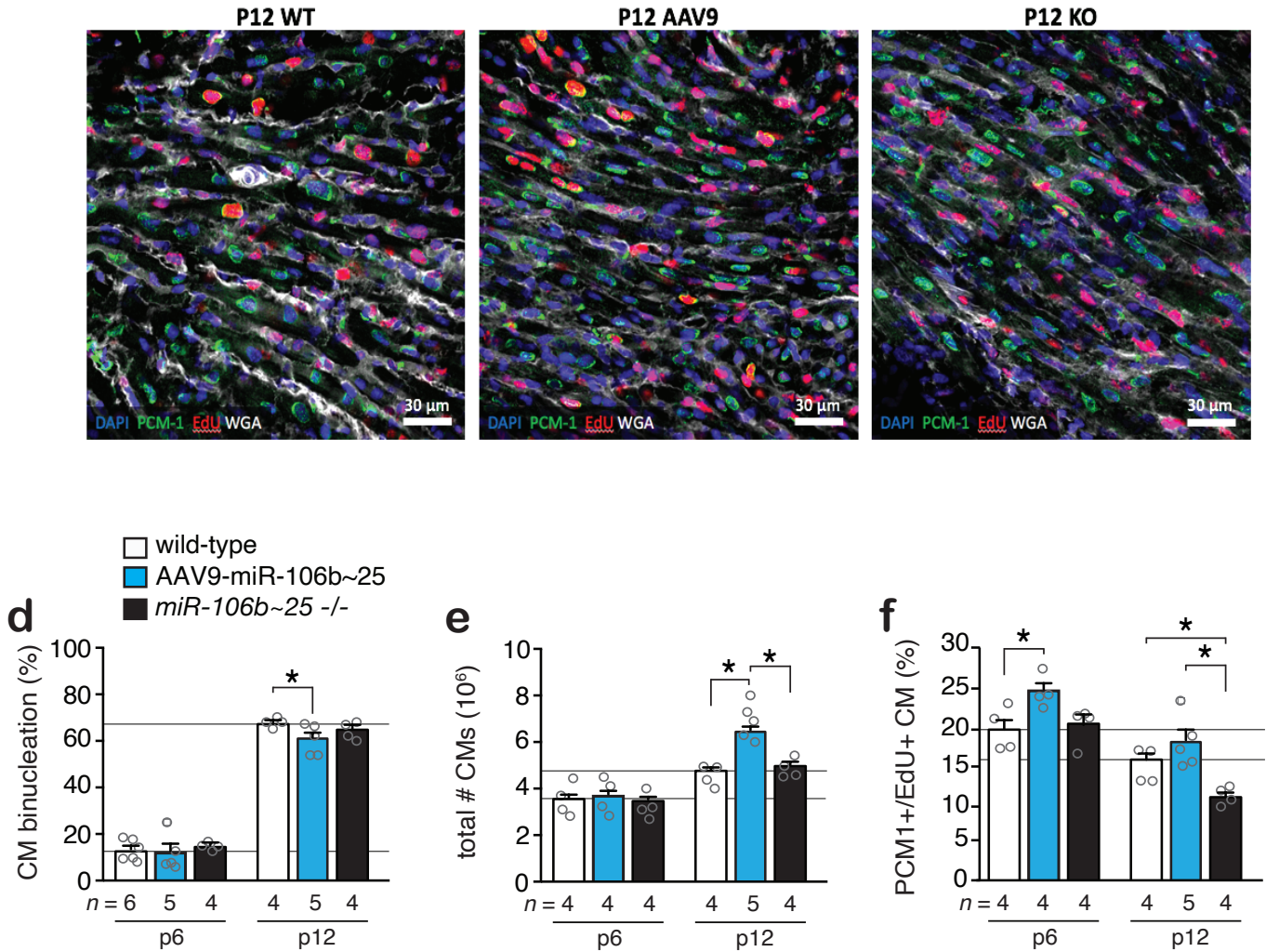
a

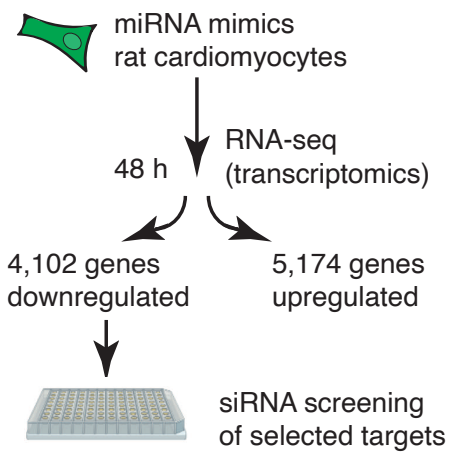

d

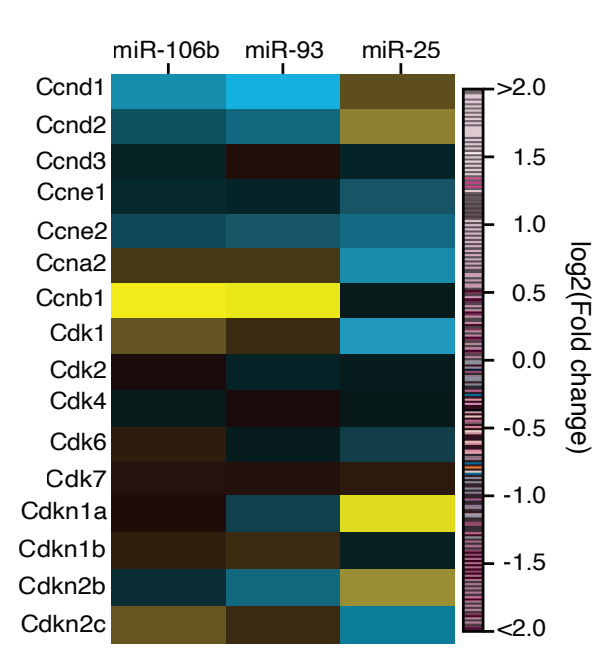

C

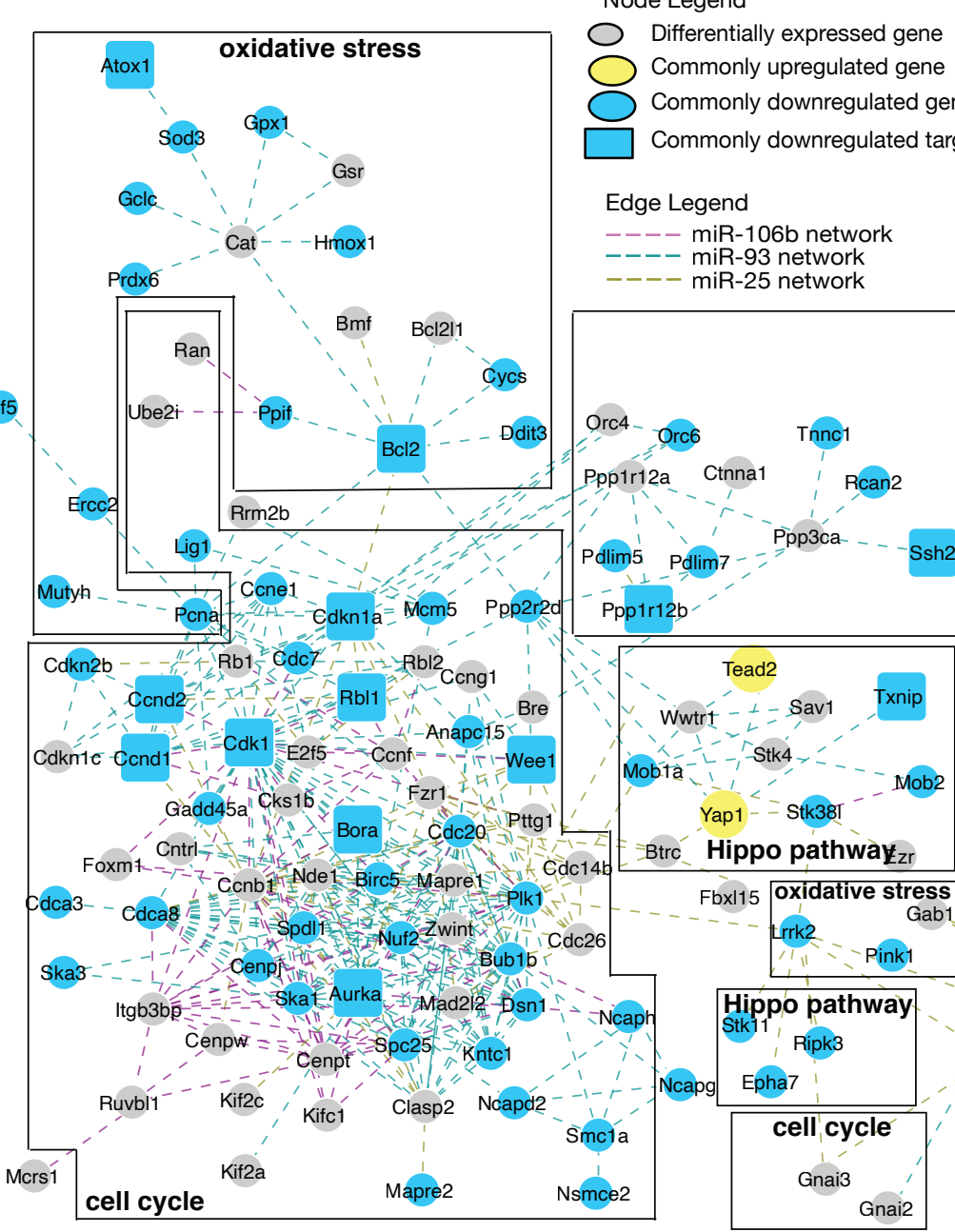

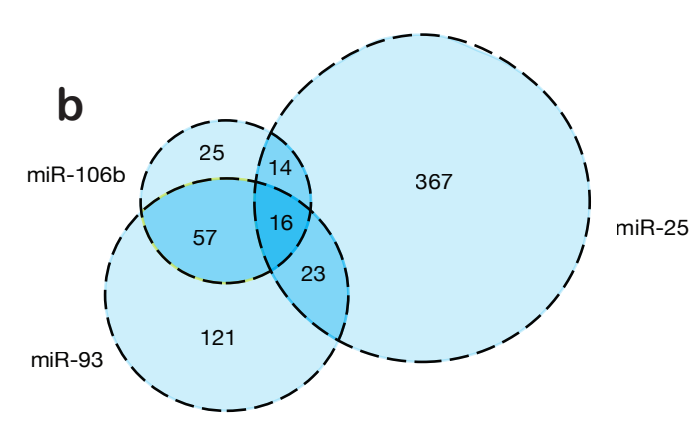

actin cytoskeleton reorganization

Coro1b
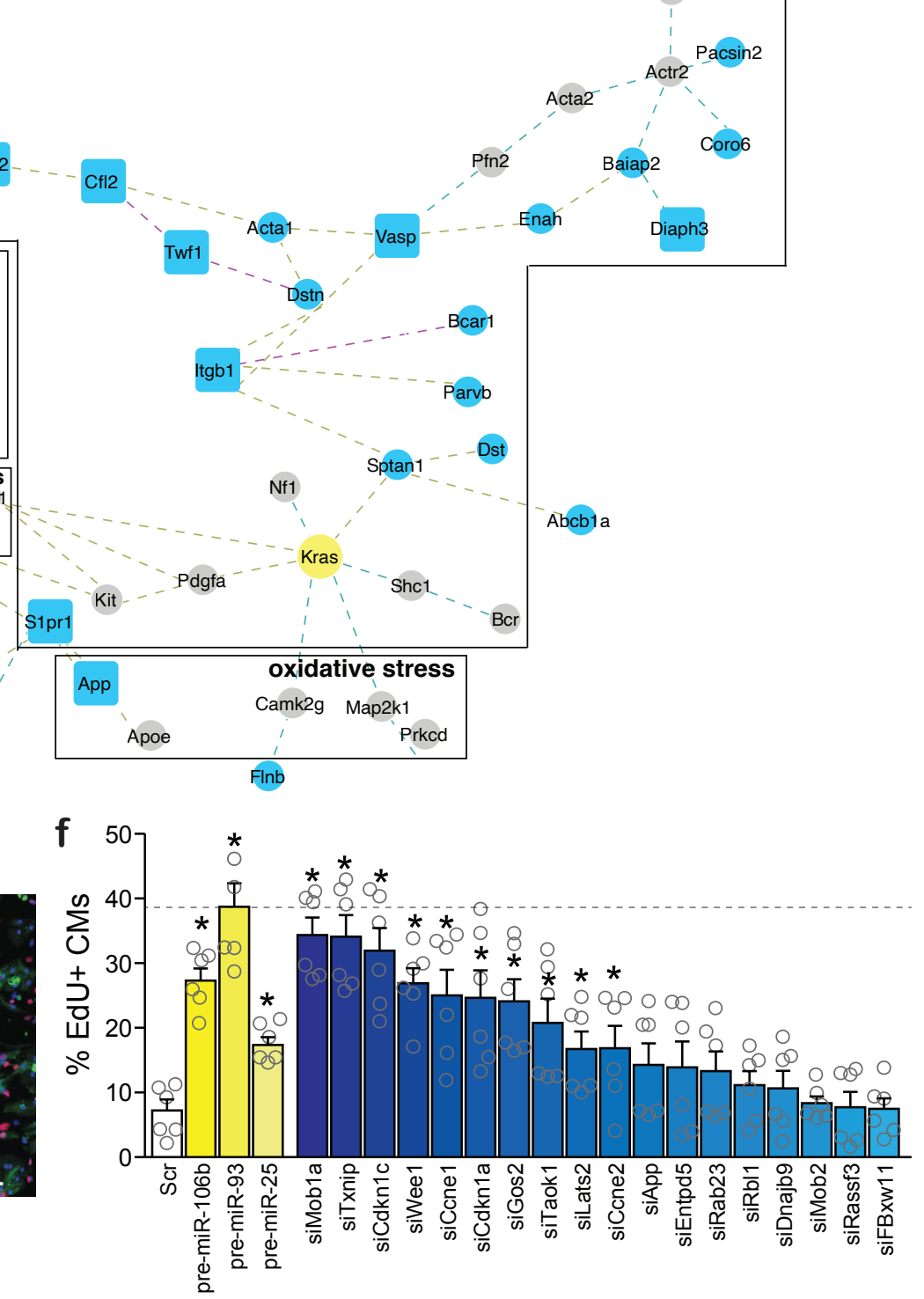

Figure 5 e

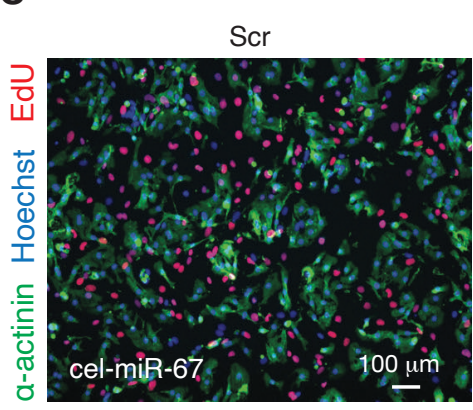

siCdkn1c

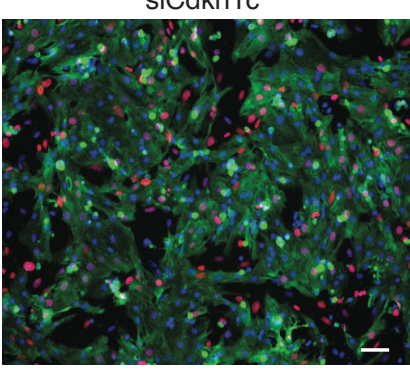

siWee1

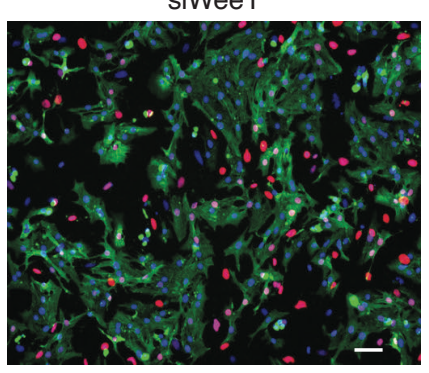

siTaok1

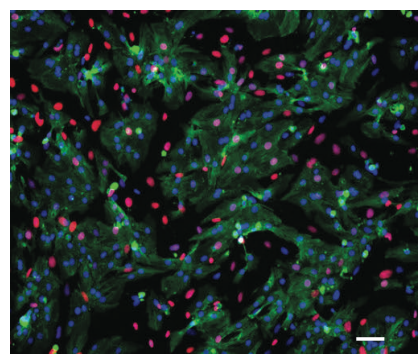


a

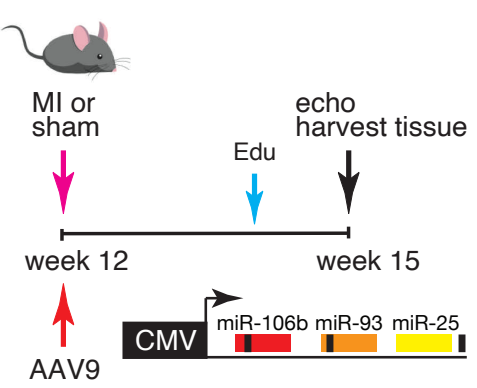

b

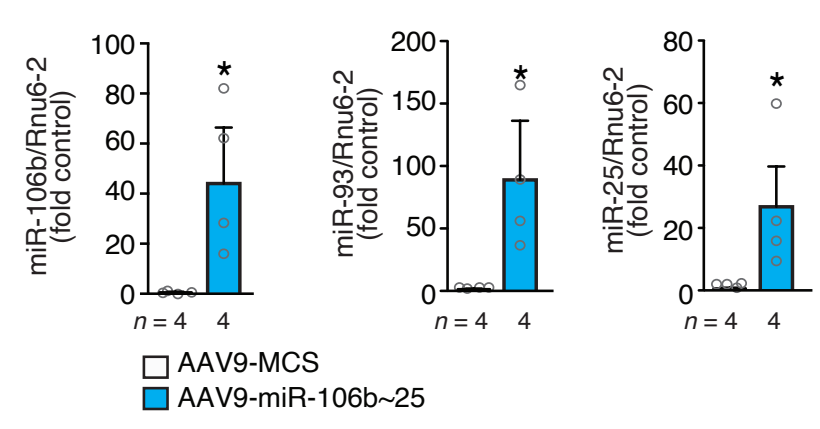

f

d

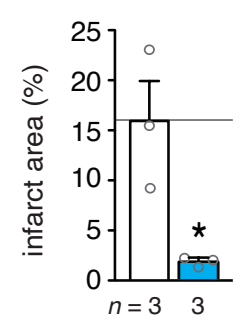

$\square$ AAV9-MCS

$\square$ AAV9-miR-106b 25

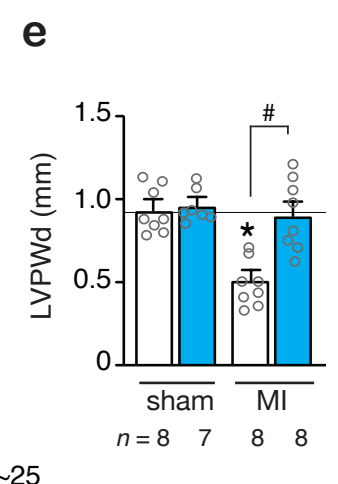

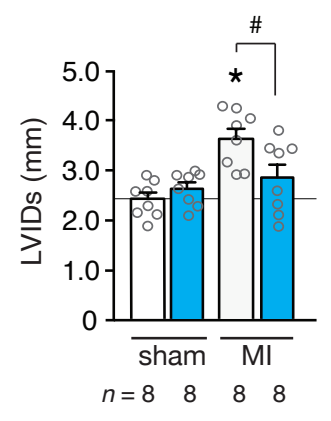

g

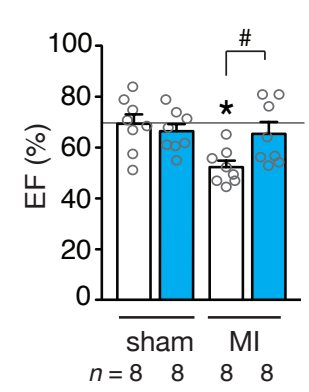

C

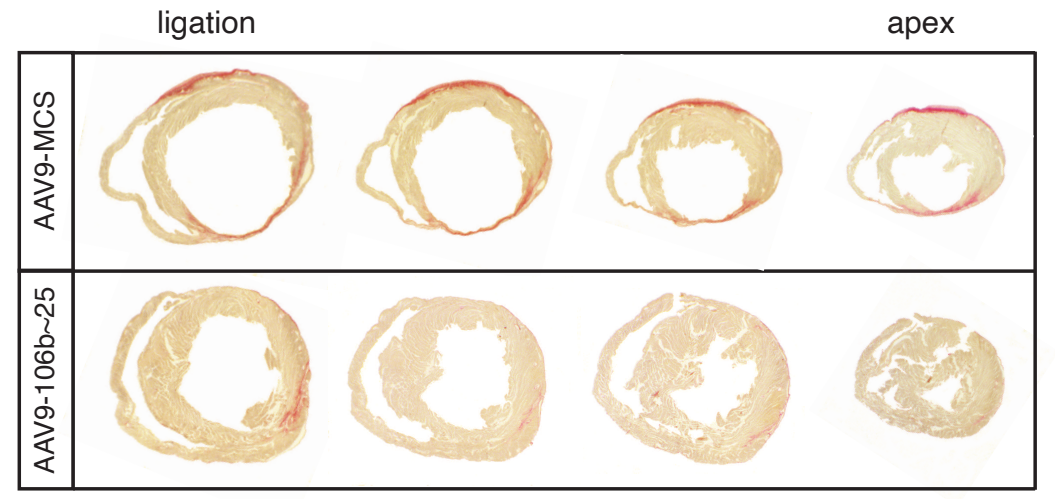

h
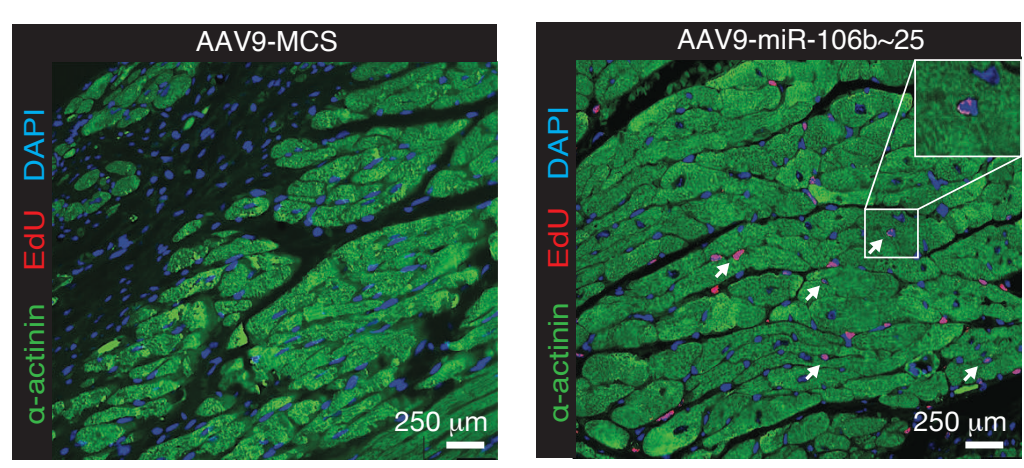

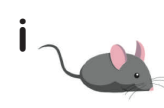

Myh6-Cre-ER ${ }^{2} \mathrm{x}$ R26-tdTomato
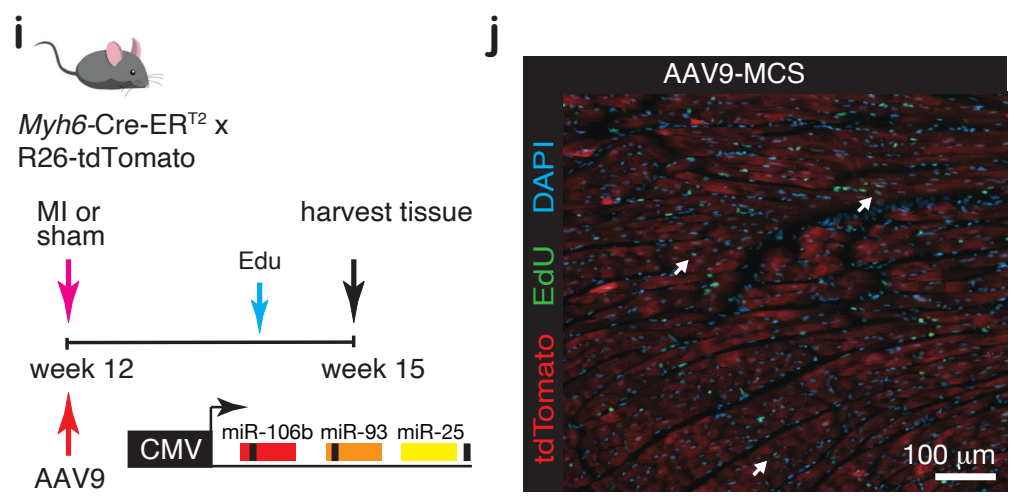

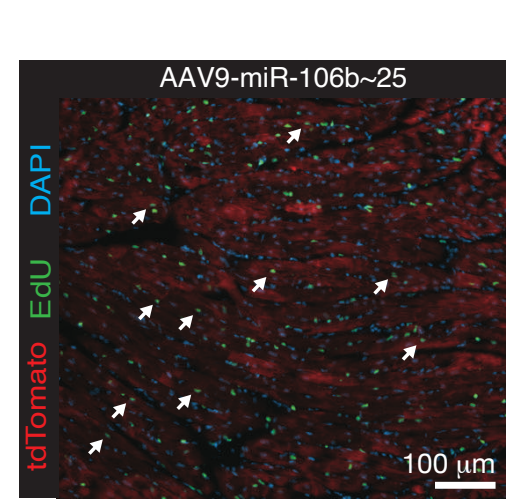

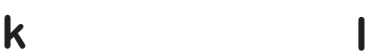
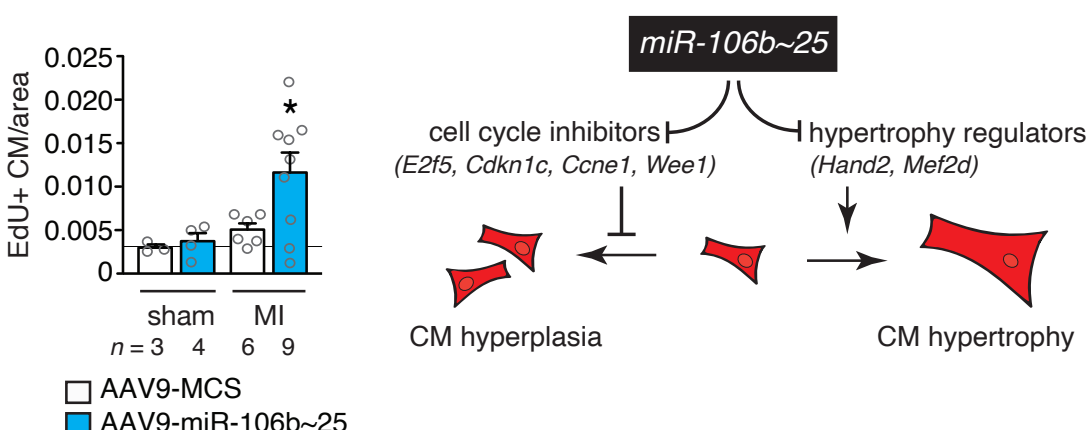